\title{
A Priori Estimates for the Free-Boundary 3D Compressible Euler Equations in Physical Vacuum
}

\author{
Daniel Coutand ${ }^{1}$, Hans Lindblad ${ }^{2}$, Steve Shkoller ${ }^{3}$ \\ 1 CANPDE, Maxwell Institute for Mathematical Sciences and Department of Mathematics, \\ Heriot-Watt University, Edinburgh, EH14 4AS, UK. E-mail: D.Coutand@ma.hw.ac.uk \\ 2 Department of Mathematics, University of California, San Diego, CA 92093, USA. \\ E-mail: lindblad@math.ucsd.edu \\ 3 Department of Mathematics, University of California, Davis, CA 95616, USA. \\ E-mail: shkoller@math.ucdavis.edu
}

Received: 10 April 2009 / Accepted: 27 December 2009

Published online: 11 March 2010 - (C) The Author(s) 2010. This article is published with open access at Springerlink.com

\begin{abstract}
We prove a priori estimates for the three-dimensional compressible Euler equations with moving physical vacuum boundary, with an equation of state given by $p(\rho)=C_{\gamma} \rho^{\gamma}$ for $\gamma>1$. The vacuum condition necessitates the vanishing of the pressure, and hence density, on the dynamic boundary, which creates a degenerate and characteristic hyperbolic free-boundary system to which standard methods of symmetrizable hyperbolic equations cannot be applied.
\end{abstract}

\section{Contents}

1. Introduction . . . . . . . . . . . . . . . . . . . . . . 559

2. Notation and Weighted Spaces . . . . . . . . . . . . . . . . . . . . 564

3. The Lagrangian Vorticity . . . . . . . . . . . . . . . . . . . . 566

4. Properties of the Determinant $J$, Cofactor Matrix $a$, Unit Normal $n$, and a Polynomial-type Inequality . . . . . . . . . . . . . 566

5. Trace Estimates and the Hodge Decomposition Elliptic Estimates . . . . . 567

6. The a priori Estimates . . . . . . . . . . . . . . . . . . . 568

7. The Case of General $\gamma>1 \ldots \ldots$. . . . . . . . . . . . . . . . . .

\section{Introduction}

1.1. The compressible Euler equations in Eulerian variables. For $0 \leq t \leq T$, the evolution of a three-dimensional compressible gas moving inside of a dynamic vacuum 
boundary is modeled by the one-phase compressible Euler equations:

$$
\begin{aligned}
\rho\left[u_{t}+u \cdot D u\right]+D p(\rho) & =0 & & \text { in } \Omega(t), \\
\rho_{t}+\operatorname{div}(\rho u) & =0 & & \text { in } \Omega(t), \\
p & =0 & & \text { on } \Gamma(t), \\
\mathcal{V}(\Gamma(t)) & =u \cdot n, & & \\
(\rho, u) & =\left(\rho_{0}, u_{0}\right) & & \text { on } \Omega(0), \\
\Omega(0) & =\Omega . & &
\end{aligned}
$$

The open, bounded subset $\Omega(t) \subset \mathbb{R}^{3}$ denotes the changing volume occupied by the gas, $\Gamma(t):=\partial \Omega(t)$ denotes the moving vacuum boundary, $\mathcal{V}(\Gamma(t))$ denotes the normal velocity of $\Gamma(t)$, and $n$ denotes the exterior unit normal vector to $\Gamma(t)$. The vector-field $u=\left(u_{1}, u_{2}, u_{3}\right)$ denotes the Eulerian velocity field, $p$ denotes the pressure function, and $\rho$ denotes the density of the gas. The equation of state $p(\rho)$ is given by

$$
p(x, t)=C_{\gamma} \rho(x, t)^{\gamma} \text { for } \gamma>1,
$$

where $C_{\gamma}$ is the adiabatic constant which we set to unity, and

$$
\rho>0 \text { in } \Omega(t) \text { and } \rho=0 \text { on } \Gamma(t) .
$$

Equation (1.1a) is the conservation of momentum; (1.1b) is the conservation of mass; the boundary condition (1.1c) states that pressure (and hence density) vanish along the vacuum boundary; (1.1d) states that the vacuum boundary is moving with the normal component of the fluid velocity, and (1.1e)-(1.1f) are the initial conditions for the density, velocity, and domain. Using the equation of state (1.2), (1.1a) is written as

$$
\rho\left[u_{t}+u \cdot D u\right]+D \rho^{\gamma}=0 \quad \text { in } \Omega(t) .
$$

1.2. Physical vacuum. With the sound speed given by $c:=\sqrt{\partial p / \partial \rho}$ and $N$ denoting the outward unit normal to $\Gamma$, satisfaction of the condition

$$
\frac{\partial c_{0}^{2}}{\partial N}<0 \text { on } \Gamma
$$

defines a physical vacuum boundary (see [10,12-15,20]), where $c_{0}=\left.c\right|_{t=0}$. The physical vacuum condition (1.3) is equivalent to the requirement that

$$
\frac{\partial \rho_{0}^{\gamma-1}}{\partial N}<0 \text { on } \Gamma \text {. }
$$

Since $\rho_{0}>0$ in $\Omega,(1.4)$ implies that for some positive constant $C$ and $x \in \Omega$ near the vacuum boundary $\Gamma$,

$$
\rho_{0}^{\gamma-1}(x) \geq C \operatorname{dist}(x, \Gamma) \text { for } x \text { near } \Gamma .
$$

Because of condition (1.5), the compressible Euler system (1.1) is a degenerate and characteristic hyperbolic system to which standard methods of symmetric hyperbolic conservation laws cannot be applied.

We note that by choosing a lower-bound with a faster rate of degeneracy such as, for example, $\operatorname{dist}(x, \Gamma(t))^{b}$ for $b=2,3, \ldots$, the analysis becomes significantly easier; 
for instance, if $b=2$, then $\frac{D \rho_{0}^{\gamma-1}(x, t)}{\sqrt{\rho_{0}^{\gamma-1}(x, t)}}$ is bounded for all $x \in \Omega$. This bound makes it possible to easily control error terms in energy estimates, and in effect removes the singular behavior associated with the physical vacuum condition (1.5).

1.3. Fixing the domain and the Lagrangian variables on $\Omega$. We transform the system (1.1) into Lagrangian variables. We let $\eta(x, t)$ denote the "position" of the gas particle $x$ at time $t$. Thus,

$$
\partial_{t} \eta=u \circ \eta \quad \text { for } t>0 \quad \text { and } \quad \eta(x, 0)=x,
$$

where $\circ$ denotes composition so that $[u \circ \eta](x, t):=u(\eta(x, t), t)$. We set

$$
\begin{aligned}
& v=u \circ \eta \text { (Lagrangian velocity), } \\
& f=\rho \circ \eta \text { (Lagrangian density), } \\
& A=[D \eta]^{-1} \text { (inverse of deformation tensor), } \\
& J=\operatorname{det} D \eta \text { (Jacobian determinant), } \\
& a=J A \text { (tranpose of cofactor matrix). }
\end{aligned}
$$

Using Einstein's summation convention defined in Sect. 2.3 below, and using the notation $F,{ }_{k}$ to denote $\frac{\partial F}{\partial x_{k}}$, the $\mathrm{k}^{t h}$-partial derivative of $F$ for $k=1,2,3$, the Lagrangian version of Eqs. (1.1a)-(1.1b) can be written on the fixed reference domain $\Omega$ as

$$
\begin{aligned}
f v_{t}^{i}+A_{i}^{k} f^{\gamma}{ }_{k} & =0 & & \text { in } \Omega \times(0, T], \\
f_{t}+f A_{i}^{j} v^{i},_{j} & =0 & & \text { in } \Omega \times(0, T], \\
f & =0 & & \text { in } \Omega \times(0, T], \\
(f, v, \eta) & =\left(\rho_{0}, u_{0}, e\right) & & \text { in } \Omega \times\{t=0\},
\end{aligned}
$$

where $e(x)=x$ denotes the identity map on $\Omega$.

Since $J_{t}=J A_{i}^{j} v^{i},{ }_{j}$, it follows that

$$
f=\rho_{0} J^{-1}
$$

so that the initial density function $\rho_{0}$ can be viewed as a parameter in the Euler equations. Let $\Gamma:=\partial \Omega$ denote the initial vacuum boundary; using that $A_{i}^{k}=J^{-1} a_{i}^{k}$, we write the compressible Euler equations (1.6) as

$$
\begin{aligned}
\rho_{0} v_{t}^{i}+a_{i}^{k}\left(\rho_{0}^{\gamma} J^{-\gamma}\right), k_{k} & =0 & & \text { in } \Omega \times(0, T], \\
(\eta, v) & =\left(e, u_{0}\right) & & \text { in } \Omega \times\{t=0\}, \\
\rho_{0}^{\gamma-1} & =0 & & \text { on } \Gamma,
\end{aligned}
$$

with $\rho_{0}^{\gamma-1}(x) \geq C \operatorname{dist}(x, \Gamma)$ for $x \in \Omega$ near $\Gamma$. 
1.4. Setting $\gamma=2$. We will focus our analysis on the case $\gamma=2$, and in Sect. 7 , we will explain the changes in the higher-order energy function for the general case of $\gamma>1$.

We seek solutions $\eta$ to the following system:

$$
\begin{aligned}
\rho_{0} v_{t}^{i}+a_{i}^{k}\left(\rho_{0}^{2} J^{-2}\right), k & =0 & & \text { in } \Omega \times(0, T], \\
(\eta, v) & =\left(e, u_{0}\right) & & \text { on } \Omega \times\{t=0\}, \\
\rho_{0} & =0 & & \text { on } \Gamma,
\end{aligned}
$$

with $\rho_{0}(x) \geq C \operatorname{dist}(x, \Gamma)$ for $x \in \Omega$ near $\Gamma$.

Equation (1.9a) is equivalent to

$$
v_{t}^{i}+2 A_{i}^{k}\left(\rho_{0} J^{-1}\right),_{k}=0
$$

and (1.10) can be written as

$$
v_{t}^{i}+\rho_{0} a_{i}^{k} J^{-2},_{k}+2 \rho_{0},{ }_{k} a_{i}^{k} J^{-2}=0 .
$$

Because of the degeneracy caused by $\rho_{0}=0$ on $\Gamma$, all three equivalent forms of the compressible Euler equations are crucially used in our analysis. Equation (1.9a) is used for energy estimates, while (1.10) is used for estimates of the vorticity, and (1.11) is used for additional elliptic-type estimates used to recover the bounds for normal derivatives.

1.5. The reference domain $\Omega$. To avoid the use of local coordinate charts necessary for arbitrary geometries, for simplicity, we will assume that the initial domain $\Omega \subset \mathbb{R}^{3}$ at time $t=0$ is given by

$$
\Omega=\left\{\left(x_{1}, x_{2}, x_{3}\right) \in \mathbb{R}^{3} \mid\left(x_{1}, x_{2}\right) \in \mathbb{T}^{2}, x_{3} \in(0,1)\right\},
$$

where $\mathbb{T}^{2}$ denotes the 2-torus, which can be thought of as the unit square with periodic boundary conditions. This permits the use of one global Cartesian coordinate system. At $t=0$, the reference vacuum boundary is the top boundary

$$
\Gamma=\left\{x_{3}=1\right\},
$$

while the bottom boundary $\left\{x_{3}=0\right\}$ is fixed with boundary condition

$$
u^{3}=0 \quad \text { on }\left\{x_{3}=0\right\} \times[0, T] .
$$

The moving vacuum boundary is then given by

$$
\Gamma(t)=\eta(t)(\Gamma)=\eta\left(x_{1}, x_{2}, 1, t\right)
$$

1.6. The higher-order energy function. For $\gamma=2$, the physical energy $\int_{\Omega}\left[\frac{1}{2} \rho_{0}|v|^{2}+\right.$ $\left.\rho_{0}^{2} J^{-1}\right] d x$ is a conserved quantity, but is far too weak for the purposes of constructing solutions; instead, we consider the higher-order energy function 


$$
\begin{aligned}
E(t)= & \sum_{a=0}^{4}\left\|\partial_{t}^{2 a} \eta(t)\right\|_{4-a}^{2}+\sum_{a=0}^{4}\left[\left\|\rho_{0} \bar{\partial}^{4-a} \partial_{t}^{2 a} D \eta(t)\right\|_{0}^{2}+\left\|\sqrt{\rho_{0}} \bar{\partial}^{4-a} \partial_{t}^{2 a} v(t)\right\|_{0}^{2}\right] \\
& +\sum_{a=0}^{3}\left\|\rho_{0} \partial_{t}^{2 a} J^{-2}(t)\right\|_{4-a}^{2}+\left\|\operatorname{curl}_{\eta} v(t)\right\|_{3}^{2}+\left\|\rho_{0} \bar{\partial}^{4} \operatorname{curl}_{\eta} v(t)\right\|_{0}^{2},
\end{aligned}
$$

where $\bar{\partial}=\left(\frac{\partial}{\partial x_{1}}, \frac{\partial}{\partial x_{2}}\right)$. Section 2 explains the notation.

While this function is not conserved, it is possible to show that $\sup _{t \in[0, T]} E(t)$ remains bounded for sufficiently smooth solutions of (1.9), whenever $T>0$ is taken sufficiently small; the bound depends only on $E(0)$.

\subsection{Main Result.}

Theorem 1.1 (The case $\gamma=2$ ). Suppose that $\eta(t)$ is a smooth solution of (1.9) on a time interval $[0, \bar{T}]$ satisfying the initial bound $E(0)<\infty$, and that the initial density function $0<\rho_{0}$ in $\Omega$ and $\rho_{0} \in H^{4}(\Omega)$ satisfies the physical vacuum condition (1.5). Then for $T>0$ taken sufficiently small, the energy function $E(t)$ constructed from the solution $\eta(t)$ satisfies the a priori estimate

$$
\sup _{t \in[0, T]} E(t) \leq M_{0}
$$

where $M_{0}$ and $T$ is a function of $E(0)$.

Of course, our theorem also covers the case that $\Omega \subset \mathbb{R}^{d}$ for $d=1$ or 2 , and by using a collection of coordinate charts, we can allow arbitrary initial domains, as long as the initial boundary is of Sobolev class $H^{3.5}$. We announced Theorem 1.1 in [4].

1.8. History of prior results for the compressible Euler equations with vacuum boundary. We are aware of only a handful of previous theorems pertaining to the existence of solutions to the compressible and inviscid Euler equations with moving vacuum boundary. Makino [16] considered compactly supported initial data, and treated the compressible Euler equations for a gas as being set on $\mathbb{R}^{3} \times(0, T]$. With his methodology, it is not possible to track the location of the vacuum boundary (nor is it necessary); nevertheless, an existence theory was developed in this context, by a variable change that permitted the standard theory of symmetric hyperbolic systems to be employed. Unfortunately, the constraints on the data are too severe to allow for the evolution of the physical vacuum boundary.

In [11], Lindblad proved existence and uniqueness for the 3D compressible Euler equations modeling a liquid rather than a gas. For a compressible liquid, the density $\rho>0$ is assumed to be a positive constant on the moving vacuum boundary $\Gamma(t)$ and is thus uniformly bounded below by a positive constant. As such, the compressible liquid provides a uniformly hyperbolic, but characteristic, system. Lindblad used Lagrangian variables combined with Nash-Moser iteration to construct solutions. More recently, Trakhinin [19] provided an alternative proof for the existence of a compressible liquid, employing a solution strategy based on symmetric hyperbolic systems combined with Nash-Moser iteration.

The only existence theory for the physical vacuum singularity that we are aware of can be found in the recent paper by Jang and Masmoudi [6] for the 1D compressible 
gas; we refer the interested reader to the introduction in that paper for a nice history of the analysis of the 1D compressible Euler equations with damping.

1.9. Generalization of the isentropic gas assumption. The general form of the compressible Euler equations in three space dimensions are the $5 \times 5$ system of conservation laws

$$
\begin{aligned}
\rho\left[u_{t}+u \cdot D u\right]+D p(\rho) & =0, \\
\rho_{t}+\operatorname{div}(\rho u) & =0, \\
(\rho \mathfrak{E})_{t}+\operatorname{div}(\rho u \mathfrak{E}+p u) & =0,
\end{aligned}
$$

where $(1.13 \mathrm{a}),(1.13 \mathrm{~b})$ and $(1.13 \mathrm{c})$ represent the respective conservation of momentum, mass, and total energy. Here, the quantity $\mathfrak{E}$ is the sum of contributions from the kinetic energy $\frac{1}{2}|u|^{2}$, and the internal energy $e$, i.e., $\mathfrak{E}=\frac{1}{2}|u|^{2}+e$. For a single phase of compressible liquid or gas, $e$ becomes a well-defined function of $\rho$ and $p$ through the theory of thermodynamics, $e=e(\rho, p)$. Other interesting and useful physical quantities, the temperature $T(\rho, p)$ and the entropy $S(\rho, p)$ are defined through the following consequence of the second law of thermodynamics

$$
T d S=d e=-\frac{p}{\rho^{2}} d \rho .
$$

For ideal gases, the quanities $e, T, S$ have the explicit formulae:

$$
\begin{aligned}
e(\rho, p) & =\frac{p}{\rho(\gamma-1)}=\frac{T}{\gamma-1}, \\
T(\rho, p) & =\frac{p}{\rho}, \\
p & =e^{S} \rho^{\gamma}, \quad \gamma>1, \text { constant. }
\end{aligned}
$$

In regions of smoothness, one often uses velocity and a convenient choice of two additional variables among the five quantities $S, T, p, \rho, e$ as independent variables. For the Lagrangian formulation, the entropy $S$ plays an important role, as it satisfies the transport equation

$$
S_{t}+(u \cdot D) S=0
$$

and as such, $S \circ \eta=S_{0}$, where $S_{0}(x)=S(x, 0)$ is the initial entropy function. Thus, by replacing $f$ with $e^{S \circ \eta} \rho_{0}^{\gamma} J^{-\gamma}$, our analysis for the isentropic case naturally generalizes to the $5 \times 5$ system of conservation laws.

\section{Notation and Weighted Spaces}

2.1. Differentiation and norms in the open set $\Omega$. The reference domain $\Omega$ is defined in Sect. 1.5. Throughout the paper the symbol $D$ will be used to denote the three-dimensional gradient vector

$$
D=\left(\frac{\partial}{\partial x_{1}}, \frac{\partial}{\partial x_{2}}, \frac{\partial}{\partial x_{3}}\right) .
$$


For integers $k \geq 0$ and a smooth, open domain $\Omega$ of $\mathbb{R}^{3}$, we define the Sobolev space $H^{k}(\Omega)\left(H^{k}\left(\Omega ; \mathbb{R}^{3}\right)\right)$ to be the completion of $C^{\infty}(\Omega)\left(C^{\infty}\left(\Omega ; \mathbb{R}^{3}\right)\right)$ in the norm

$$
\|u\|_{k}:=\left(\sum_{|a| \leq k} \int_{\Gamma}\left|\frac{\partial^{|a|} u(x)}{\partial x_{1}^{a_{1}} \partial x_{2}^{a_{2}} \partial x_{3}^{a_{3}}}\right|^{2} d x_{1} d x_{2} d x_{3}\right)^{1 / 2},
$$

for a multi-index $a \in \mathbb{Z}_{+}^{3}$, with the standard convention that $|a|=a_{1}+a_{2}+a_{3}$. For real numbers $s \geq 0$, the Sobolev spaces $H^{s}(\Omega)$ and the norms $\|\cdot\|_{s}$ are defined by interpolation. We will write $H^{s}(\Omega)$ instead of $H^{s}\left(\Omega ; \mathbb{R}^{3}\right)$ for vector-valued functions. In the case that $s \geq 3$, the above definition also holds for domains $\Omega$ of class $H^{s}$. We will write $d x$ to denote the 3 -D Lebesgue measure $d x_{1} d x_{2} d x_{3}$.

2.2. Tangent and normal vectors to $\Gamma$. The outward-pointing unit normal vector to $\Gamma$ is given by

$$
N=(0,0,1) .
$$

Similarly, the unit tangent vectors on $\Gamma$ are given by

$$
T_{1}=(1,0,0) \text { and } T_{2}=(0,1,0) .
$$

2.3. Einstein's summation convention. Repeated Latin indices $i, j, k$, etc., are summed from 1 to 3, and repeated Greek indices $\alpha, \beta, \gamma$, etc., are summed from 1 to 2 . For example, $F,{ }_{i i}:=\sum_{i=1,3} \frac{\partial^{2}}{\partial x_{i} \partial x_{i}}$, and $F^{i},{ }_{\alpha} I^{\alpha \beta} G^{i}, \beta=\sum_{i=1}^{3} \sum_{\alpha=1}^{2} \sum_{\beta=1}^{2} \frac{\partial F^{i}}{\partial x_{\alpha}} I^{\alpha \beta} \frac{\partial G^{i}}{\partial x_{\beta}}$.

2.4. Sobolev spaces on $\Gamma$. For functions $u \in H^{k}(\Gamma), k \geq 0$, we set

$$
|u|_{k}:=\left(\sum_{|a| \leq k} \int_{\Omega}\left|\frac{\partial^{|\alpha|} u(x)}{\partial x_{1}^{\alpha_{1}} \partial x_{2}^{\alpha_{2}}}\right|^{2} d x_{1} d x_{2}\right)^{1 / 2}
$$

for a multi-index $\alpha \in \mathbb{Z}_{+}^{2}$. For real $s \geq 0$, the Hilbert space $H^{s}(\Gamma)$ and the boundary norm $|\cdot|_{s}$ is defined by interpolation. The negative-order Sobolev spaces $H^{-s}(\Gamma)$ are defined via duality: for real $s \geq 0$,

$$
H^{-s}(\Gamma):=\left[H^{s}(\Gamma)\right]^{\prime} .
$$

2.5. Notation for derivatives and norms. Throughout the paper, we will use the following notation:

$$
\begin{aligned}
D & =\text { three-dimensional gradient vector }=\left(\frac{\partial}{\partial x_{1}}, \frac{\partial}{\partial x_{2}}, \frac{\partial}{\partial x_{3}}\right), \\
\bar{\partial} & =\text { two-dimensional gradient vector or horizontal derivative }=\left(\frac{\partial}{\partial x_{1}}, \frac{\partial}{\partial x_{2}}\right), \\
\|\cdot\|_{s} & =H^{s}(\Omega) \text { interior norm, } \\
|\cdot|_{s} & =H^{s}(\Gamma) \text { boundary norm. }
\end{aligned}
$$

The $k^{\text {th }}$ partial derivative of $F$ will be denoted by $F,_{k}=\frac{\partial F}{\partial x_{k}}$. 
2.6. The embedding of a weighted Sobolev space. Using $d$ to denote the distance function to the boundary $\Gamma$, and letting $p=1$ or 2 , the weighted Sobolev space $H_{d^{p}}^{1}(\Omega)$, with norm given by $\int_{\Omega} d(x)^{p}\left(|F(x)|^{2}+|D F(x)|^{2}\right) d x$ for any $F \in H_{d^{p}}^{1}(\Omega)$, satisfies the following embedding:

$$
H_{d^{p}}^{1}(\Omega) \hookrightarrow H^{1-\frac{p}{2}}(\Omega),
$$

so that there is a constant $C>0$ depending only on $\Omega$ and $p$ such that

$$
\|F\|_{1-p / 2}^{2} \leq C \int_{\Omega} d(x)^{p}\left(|F(x)|^{2}+|D F(x)|^{2}\right) d x .
$$

See, for example, Sect. 8.8 in Kufner [9].

\section{The Lagrangian Vorticity}

We make use of the permutation symbol

$$
\varepsilon_{i j k}=\left\{\begin{aligned}
1, & \text { even permutation of }\{1,2,3\}, \\
-1, & \text { odd permutation of }\{1,2,3\}, \\
0, & \text { otherwise, }
\end{aligned}\right.
$$

and the basic identity regarding the $i^{\text {th }}$ component of the curl of a vector field $u$ :

$$
(\operatorname{curl} u)_{i}=\varepsilon_{i j k} u^{k},{ }_{j} .
$$

The chain rule shows that

$$
(\operatorname{curl} u(\eta))_{i}=\left(\operatorname{curl}_{\eta} v\right)_{i}:=\varepsilon_{i j k} A_{j}^{s} v^{k}, s,
$$

the right-hand side defining the Lagrangian curl operator $\operatorname{curl}_{\eta}$. Taking the Lagrangian curl of (1.10) yields the Lagrangian vorticity equation

$$
\varepsilon_{k j i} A_{j}^{s} v_{t}^{i}, s_{s}=0, \quad \text { or } \quad \operatorname{curl}_{\eta} v_{t}=0 .
$$

\section{Properties of the Determinant $J$, Cofactor Matrix $a$, Unit Normal $n$, and a Polynomial-type Inequality}

4.1. Differentiating the Jacobian determinant. The following identities will be useful to us:

$$
\begin{aligned}
\bar{\partial} J & \left.=a_{r}^{s} \bar{\partial} \frac{\partial \eta^{r}}{\partial x^{s}} \text { (horizontal differentiation }\right), \\
\partial_{t} J & =a_{r}^{s} \frac{\partial v^{r}}{\partial x^{s}} \quad\left(\text { time differentiation using } v=\eta_{t}\right) .
\end{aligned}
$$

4.2. Differentiating the cofactor matrix. Using (4.1) and (4.2) and the fact that $a=J A$, we find that

$$
\begin{aligned}
\bar{\partial} a_{i}^{k} & =\bar{\partial} \frac{\partial \eta^{r}}{\partial x^{s}} J^{-1}\left[a_{r}^{s} a_{i}^{k}-a_{i}^{s} a_{r}^{k}\right] \text { (horizontal differentiation), } \\
\partial_{t} a_{i}^{k} & =\frac{\partial v^{r}}{\partial x^{s}} J^{-1}\left[a_{r}^{s} a_{i}^{k}-a_{i}^{s} a_{r}^{k}\right] \text { (time differentiation using } v=\eta_{t} \text { ). }
\end{aligned}
$$


4.3. The Piola identity. It is a fact that the columns of every cofactor matrix are divergence-free and satisfy

$$
a_{i}^{k}, k=0 .
$$

The identity (4.5) will play a vital role in our energy estimates. (Note that we use the notation cofactor for what is commonly termed the adjugate matrix, or the transpose of the cofactor.)

4.4. Geometric identities. The vectors $\eta,{ }_{\alpha}$ for $\alpha=1,2$ span the tangent plane to the surface $\Gamma$ in $\mathbb{R}^{3}$, and

$$
\tau_{1}:=\frac{\eta, 1}{|\eta, 1|}, \quad \tau_{2}:=\frac{\eta, 2}{|\eta, 2|}, \quad \text { and } \quad n:=\frac{\eta, 1 \times \eta, 2}{|\eta, 1 \times \eta, 2|}
$$

are the unit tangent and normal vectors, respectively, to $\eta(\Gamma)$.

By definition of the cofactor matrix,

$$
a_{i}^{3}=\left[\begin{array}{cccc}
\eta^{2},{ }_{1} & \eta^{3}, 2 & -\eta^{3},{ }_{1} & \eta^{2}, 2 \\
\eta^{3},{ }_{1} & \eta^{1}, 2 & -\eta^{1},{ }_{1} & \eta^{3}, 2 \\
\eta^{1},{ }_{1} & \eta^{2}, 2 & -\eta^{1}, 2 & \eta^{2}, 1
\end{array}\right] .
$$

4.5. A polynomial-type inequality. For a constant $M_{0} \geq 0$, suppose that $f(t) \geq 0$, $t \mapsto f(t)$ is continuous, and

$$
f(t) \leq M_{0}+C t P(f(t)),
$$

where $P$ denotes a polynomial function, and $C$ is a generic constant. Then for $t$ taken sufficiently small, we have the bound

$$
f(t) \leq 2 M_{0} .
$$

This type of inequality, which we introduced in [2], can be viewed as a generalization of standard nonlinear Gronwall inequalities. (4.7).

With $E(t)$ defined by (1.12), we will show that $\sup _{t \in[0, T]} E(t)$ satisfies the inequality

\section{Trace Estimates and the Hodge Decomposition Elliptic Estimates}

The normal trace theorem which states that the existence of the normal trace of a velocity field $w \in L^{2}(\Omega)$ relies on the regularity of $\operatorname{div} w$ (see, for example, [18]). If $\operatorname{div} w \in$ $H^{1}(\Omega)^{\prime}$, then $w \cdot N$, the normal trace, exists in $H^{-0.5}(\Gamma)$ so that

$$
\|w \cdot N\|_{H^{-0.5}(\Gamma)}^{2} \leq C\left[\|w\|_{L^{2}(\Omega)}^{2}+\|\operatorname{div} w\|_{H^{1}(\Omega)^{\prime}}^{2}\right]
$$

for some constant $C$ independent of $w$. In addition to the normal trace theorem, we have the following.

Lemma 5.1. Let $w \in L^{2}(\Omega)$ so that $\operatorname{curl} w \in H^{1}(\Omega)^{\prime}$, and let $T_{1}, T_{2}$ denote the unit tangent vectors on $\Gamma$, so that any vector field $u$ on $\Gamma$ can be uniquely written as $u^{\alpha} T_{\alpha}$. Then 


$$
\left\|w \cdot T_{\alpha}\right\|_{H^{-0.5}(\Gamma)}^{2} \leq C\left[\|w\|_{L^{2}(\Omega)}^{2}+\|\operatorname{curl} w\|_{H^{1}(\Omega)^{\prime}}^{2}\right], \quad \alpha=1,2
$$

for some constant $C$ independent of $w$.

See [1] for the proof. Combining (5.1) and (5.2),

$$
\|w\|_{H^{-0.5}(\Gamma)} \leq C\left[\|w\|_{L^{2}(\Omega)}+\|\operatorname{div} w\|_{H^{1}(\Omega)^{\prime}}+\|\operatorname{curl} w\|_{H^{1}(\Omega)^{\prime}}\right]
$$

for some constant $C$ independent of $w$.

The construction of our higher-order energy function is based on the following Hodgetype elliptic estimate:

Proposition 5.2. For an $H^{r}$ domain $\Omega, r \geq 3$, if $F \in L^{2}\left(\Omega ; \mathbb{R}^{3}\right)$ with curl $F \in$ $H^{s-1}\left(\Omega ; \mathbb{R}^{3}\right)$, $\operatorname{div} F \in H^{s-1}(\Omega)$, and $\left.F \cdot N\right|_{\Gamma} \in H^{s-\frac{1}{2}}(\Gamma)$ for $1 \leq s \leq r$, then there exists a constant $\bar{C}>0$ depending only on $\Omega$ such that

$$
\begin{gathered}
\|F\|_{s} \leq \bar{C}\left(\|F\|_{0}+\|\operatorname{curl} F\|_{s-1}+\|\operatorname{div} F\|_{s-1}+|\bar{\partial} F \cdot N|_{s-\frac{3}{2}}\right), \\
\|F\|_{s} \leq \bar{C}\left(\|F\|_{0}+\|\operatorname{curl} F\|_{s-1}+\|\operatorname{div} F\|_{s-1}+\sum_{\alpha=1}^{2}\left|\bar{\partial} F \cdot T_{\alpha}\right|_{s-\frac{3}{2}}\right),
\end{gathered}
$$

where $N$ denotes the outward unit-normal to $\Gamma$, and $T_{\alpha}$ are tangent vectors for $\alpha=1,2$. These estimates are well-known and follows from the identity $-\Delta F=\operatorname{curl} \operatorname{curl} F-$ $D \operatorname{div} F$; a convenient reference is Taylor [17].

\section{The a priori Estimates}

6.1. Curl Estimates. Following Lemma 10.1 in [3], we obtain the following estimates:

Proposition 6.1. For all $t \in(0, T)$,

$$
\sum_{a=0}^{3}\left\|\operatorname{curl} \partial_{t}^{2 a} \eta(t)\right\|_{3-a}^{2}+\sum_{l=0}^{4}\left\|\rho_{0} \bar{\partial}^{4-l} \operatorname{curl} \partial_{t}^{2 l} \eta(t)\right\|_{0}^{2} \leq M_{0}+C T P\left(\sup _{t \in[0, T]} E(t)\right) .
$$

Proof. From (3.1), $\left(\operatorname{curl}_{\eta} v\right)_{t}^{k}=\varepsilon_{k j i} A_{t}{ }_{j}^{s} v^{i},{ }_{s}=: B(A, D v)$, where $B$ is quadratic in its arguments; hence,

$$
\operatorname{curl}_{\eta} v(t)=\operatorname{curl} u_{0}+\int_{0}^{t} B\left(A\left(t^{\prime}\right), D v\left(t^{\prime}\right)\right) d t^{\prime},
$$

and computing the gradient of this relation yields

$$
D \operatorname{curl}_{\eta} v(t)=\operatorname{curl} D u_{0}-\varepsilon_{\cdot j i} D A_{j}^{s} v^{i},{ }_{s}+\int_{0}^{t} D B\left(A\left(t^{\prime}\right), D v\left(t^{\prime}\right)\right) d t^{\prime} .
$$

Applying the fundamental theorem of calculus once again, shows that

$$
\begin{aligned}
D \operatorname{curl}_{\eta} \eta(t)= & t D \operatorname{curl} u_{0}+\varepsilon_{\cdot j i} \int_{0}^{t}\left[A_{t j}^{s} D \eta^{i},{ }_{s}-D A_{j}^{s} v^{i}, s\right] d t^{\prime} \\
& +\int_{0}^{t} \int_{0}^{t^{\prime}} D B\left(A\left(t^{\prime \prime}\right), D v\left(t^{\prime \prime}\right)\right) d t^{\prime \prime} d t^{\prime}
\end{aligned}
$$


and finally that

$$
\begin{aligned}
\operatorname{curl} D \eta(t)= & t \operatorname{curl} D u_{0}-\varepsilon \cdot j i \int_{0}^{t} A_{t}{ }_{j}\left(t^{\prime}\right) d t^{\prime} D \eta^{i}, s \\
& +\varepsilon_{\cdot j i} \int_{0}^{t}\left[A_{t}{ }_{j}^{s} D \eta^{i},{ }_{s}-D A_{j}^{s} v^{i},{ }_{s}\right] d t^{\prime}+\int_{0}^{t} \int_{0}^{t^{\prime}} D B\left(A\left(t^{\prime \prime}\right), D v\left(t^{\prime \prime}\right)\right) d t^{\prime \prime} d t^{\prime} .
\end{aligned}
$$

To obtain an estimate for $\|\operatorname{curl} \eta(t)\|_{3}^{2}$, we let $D^{2}$ act on (6.3). With $\partial_{t} A_{j}^{s}=$ $-A_{l}^{s} v^{l},{ }_{p} A_{j}^{p}$ and $D A_{j}^{s}=-A_{l}^{s} D \eta^{l}{ }_{, p} A_{j}^{p}$, we see that the first three terms on the righthand side of (6.3) are bounded by $M_{0}+C T P\left(\sup _{t \in[0, T]} E(t)\right)$, where we remind the reader that $M_{0}=P(E(0))$ is a polynomial function of the $E$ at time $t=0$. Since

$$
D B(A, D v)=-\varepsilon_{k j i}\left[D v^{i},{ }_{s} A_{l}^{s} v^{l},{ }_{p} A_{j}^{p}+v^{i},_{s} A_{l}^{s} D v^{l},{ }_{p} A_{j}^{p}+v^{i}{ }_{s} v^{l},{ }_{p} D\left(A_{l}^{s} A_{j}^{p}\right)\right],
$$

the highest-order term arising from the action of $D^{2}$ on $D B(A, D v)$ is written as

$$
-\varepsilon_{k j i} \int_{0}^{t} \int_{0}^{t^{\prime}}\left[D^{3} v^{i},{ }_{s} A_{l}^{s} v^{l},{ }_{p} A_{j}^{p}+v^{i},{ }_{s} A_{l}^{s} D^{3} v^{l},{ }_{p} A_{j}^{p}\right] d t^{\prime \prime} d t^{\prime} .
$$

Both summands in the integrand scale like $D^{3} v D v A A$. The precise structure of this summand is not very important; rather, the derivative count is the focus. Integrating by parts in time,

$\int_{0}^{t} \int_{0}^{t^{\prime}} D^{3} v D v A A d t^{\prime \prime} d t^{\prime}=-\int_{0}^{t} \int_{0}^{t^{\prime}} D^{3} \eta(D v A A)_{t} d t^{\prime \prime} d t^{\prime}+\int_{0}^{t} D^{3} \eta D v A A d t^{\prime}$, from which it follows that

$$
\left\|\int_{0}^{t} \int_{0}^{t^{\prime}} D^{3} B\left(A\left(t^{\prime \prime}\right), D v\left(t^{\prime \prime}\right)\right) d t^{\prime \prime} d t^{\prime}\right\|_{0}^{2} \leq C T P\left(\sup _{t \in[0, T]} E(t)\right),
$$

and hence

$$
\sup _{t \in[0, T]}\|\operatorname{curl} \eta(t)\|_{3}^{2} \leq M_{0}+C T P\left(\sup _{t \in[0, T]} E(t)\right) .
$$

Next, we show that

$$
\left\|\operatorname{curl} v_{t}(t)\right\|_{2}^{2} \leq M_{0}+C T P\left(\sup _{t \in[0, T]} E(t)\right)
$$

From (3.1),

$$
\operatorname{curl} v_{t}=\varepsilon_{j \cdot i} \int_{0}^{t} A_{t}^{s}\left(t^{\prime}\right) d t^{\prime} v_{t}^{i}, s .
$$

Since $H^{2}(\Omega)$ is a multiplicative algebra, we can directly estimate the $H^{2}(\Omega)$-norm of curl $v_{t}$ to prove that (6.4) holds. The estimates for curl $v_{t t t}(t)$ in $H^{1}(\Omega)$ and curl $\partial_{t}^{5} v(t)$ in $L^{2}(\Omega)$ follow the same argument.

The weighted estimates follow from similar reasoning. We first show that

$$
\left\|\rho_{0} \bar{\partial}^{4} \operatorname{curl} \eta(t)\right\|_{0}^{2} \leq M_{0}+C T P\left(\sup _{t \in[0, T]} E(t)\right) .
$$


To prove this weighted estimate, we write (6.2) as

$$
\operatorname{curl} v(t)=\varepsilon_{j k i} v^{i}, \int_{0}^{t} A_{t}^{s}\left(t^{\prime}\right) d t^{\prime}+\operatorname{curl} u_{0}+\int_{0}^{t} B\left(A\left(t^{\prime}\right), D v\left(t^{\prime}\right)\right) d t^{\prime},
$$

and integrate in time to find that

$$
\operatorname{curl} \eta(t)=t \operatorname{curl} u_{0}+\int_{0}^{t} \varepsilon_{j k i} v^{i}, \int_{0}^{t^{\prime}} A_{t}^{s}\left(t^{\prime \prime}\right) d t^{\prime \prime} d t^{\prime}+\int_{0}^{t} \int_{0}^{t^{\prime}} B\left(A\left(t^{\prime \prime}\right), D v\left(t^{\prime \prime}\right)\right) d t^{\prime \prime} d t^{\prime} .
$$

It follows that

$$
\begin{aligned}
& \rho_{0} \bar{\partial}^{4} \operatorname{curl} \eta(t)=t \rho_{0} \bar{\partial}^{4} \operatorname{curl} u_{0} \\
& +\int_{0}^{t} \int_{0}^{t^{\prime}} \varepsilon_{k j i} A_{t}{ }_{j} \rho_{0} \bar{\partial}^{4} v^{i},{ }_{s} d t^{\prime \prime} d t^{\prime}+\int_{0}^{t} \int_{0}^{t^{\prime}} \varepsilon_{k j i} \rho_{0} \bar{\partial}^{4} A_{t}{ }_{j}^{s} v^{i},_{s} d t^{\prime \prime} d t^{\prime} \\
& \quad+\int_{0}^{t} \varepsilon_{j k i} \rho_{0} \bar{\partial}^{4} v^{i},{ }_{s} \int_{0}^{t^{\prime}} A_{t}{ }_{j}^{s}\left(t^{\prime \prime}\right) d t^{\prime \prime} d t^{\prime}+\int_{0}^{t} \varepsilon_{j k i} v^{i},{ }_{s} \int_{0}^{t^{\prime}} \rho_{0} \bar{\partial}^{4} A_{t}{ }_{j}\left(t^{\prime \prime}\right) d t^{\prime \prime} d t^{\prime}+\mathfrak{R}_{2},
\end{aligned}
$$

where $\mathfrak{R}_{2}$ denotes terms which are lower-order in the derivative count; in particular the terms with the highest derivative count in $\Re_{2}$ scale like $\rho \bar{\partial}^{3} D v$ or $\rho \bar{\partial}^{4} \eta$, and hence satisfy the inequality $\left\|\Re_{2}(t)\right\|_{0}^{2} \leq M_{0}+C T P\left(\sup _{t \in[0, T]} E(t)\right)$. We focus on the first integral on the right-hand side of (6.6); integrating by parts in time, we find that

$$
\begin{aligned}
\int_{0}^{t} \int_{0}^{t^{\prime}} \varepsilon_{k j i} A_{t j}^{s} \rho_{0} \bar{\partial}^{4} v^{i},_{s} d t^{\prime \prime} d t^{\prime}= & -\int_{0}^{t} \int_{0}^{t^{\prime}} \varepsilon_{k j i} A_{t t}{ }_{j} \rho_{0} \bar{\partial}^{4} \eta^{i},_{s} d t^{\prime \prime} d t^{\prime} \\
& +\int_{0}^{t} \varepsilon_{k j i} A_{t}{ }_{j}^{s} \rho_{0} \bar{\partial}^{4} \eta^{i},_{s} d t^{\prime}
\end{aligned}
$$

and hence

$$
\left\|\int_{0}^{t} \int_{0}^{t^{\prime}} \varepsilon_{k j i} A_{t}{ }_{j}^{s} \rho_{0} \bar{\partial}^{4} v^{i}{ }_{s} d t^{\prime \prime} d t^{\prime}\right\|_{0}^{2} \leq M_{0}+C T P\left(\sup _{t \in[0, T]} E(t)\right) .
$$

The other time integrals in (6.6) can be estimated in the same fashion, which proves that (6.5) holds. The weighted estimates for the curl of $v_{t}, v_{t t t}$ and $\partial_{t}^{5} v$ are obtained similarly.

6.2. Energy estimates. We assume that we have smooth solutions $\eta$ on a time interval $[0, T]$, and that for all such solutions, the time $T>0$ is taken sufficiently small so that for $t \in[0, T]$,

$$
\begin{aligned}
\frac{1}{2} \leq J(t) & \leq \frac{3}{2} \\
\|\eta(t)\|_{3.5}^{2} & \leq 2\|e\|_{3.5}^{2}+1 \\
\left\|\partial_{t}^{a} v(t)\right\|_{3-a / 2}^{2} & \leq 2\left\|\partial_{t}^{a} v(0)\right\|_{3-a / 2}^{2}+1 \quad \text { for } a=0,1, \ldots, 6 .
\end{aligned}
$$

The right-hand sides appearing in these inequalities shall be denoted by a generic constant $C$ in the estimates appearing below. Once we establish our a priori bounds, we can ensure that our solution verifies to the assumptions (6.7) by means of the fundamental theorem of calculus. 
6.2.1. The structure of the estimates. Due to the degeneracy of the initial density function $\rho_{0}$, one time derivative scales like one-half of a space derivative. The energy estimates for the time and tangential derivatives are obtained by first studying the $\bar{\partial}^{4}$-differentiated Euler equations, then the $\bar{\partial}^{3} \partial_{t}^{2}$-differentiated Euler equations, and so on, until we reach the $\bar{\partial}^{0} \partial_{t}^{8}$-differentiated Euler equations. The estimates for the normal derivatives are then found using elliptic-type estimates. The Sobolev embedding theorem requires that we use $H^{4}(\Omega)$ as the minimal regularity of $\eta(t)$.

\subsubsection{The $\bar{\partial}^{4}$-problem.}

Proposition 6.2. For $\delta>0$ and letting the constant $M_{0}$ depend on $1 / \delta$,

$$
\begin{aligned}
& \sup _{t \in[0, T]}\left(\int_{\Omega} \rho_{0}(x)\left|\bar{\partial}^{4} v(x, t)\right|^{2} d x+\int_{\Omega} \rho_{0}^{2}(x)\left|\bar{\partial}^{4} D \eta(x, t)\right|^{2} d x\right) \\
& \quad \leq M_{0}+\delta \sup _{t \in[0, T]} E(t)+C T P\left(\sup _{t \in[0, T]} E(t)\right) .
\end{aligned}
$$

Proof. Letting $\bar{\partial}^{4}$ act on $\rho_{0} v_{t}^{i}+a_{i}^{k}\left(\rho_{0}^{2} J^{-2}\right),{ }_{k}=0$, and taking the $L^{2}(\Omega)$-inner product with $\bar{\partial}^{4} v^{i}$, we obtain

$$
\begin{aligned}
& \frac{1}{2} \frac{d}{d t} \int_{\Omega} \rho_{0}\left|\bar{\partial}^{4} v\right|^{2} d x+\int_{\Omega} \bar{\partial}^{4} a_{i}^{k}\left(\rho_{0}^{2} J^{-2}\right),{ }_{k} \bar{\partial}^{4} v^{i} d x+\int_{\Omega} a_{i}^{k}\left(\rho_{0}^{2} \bar{\partial}^{4} J^{-2}\right), \bar{\partial}^{4} v^{i} d x \\
& \quad+\int_{\Omega} a_{i}^{k}\left(\bar{\partial}^{4} \rho_{0}^{2} J^{-2}\right),_{k} \bar{\partial}^{4} v^{i} d x=\sum_{l=1}^{3} c_{l} \int_{\Omega} \bar{\partial}^{4-l} a_{i}^{k}\left(\rho_{0}^{2} \bar{\partial}^{l} J^{-2}\right),_{k} \bar{\partial}^{4} v^{i} d x .
\end{aligned}
$$

Integrating the first term from 0 to $t \in(0, T]$ produces the first term on the left-hand side of (6.8).

We define the following integrals:

$$
\begin{aligned}
& \mathcal{I}_{1}=\int_{\Omega} \bar{\partial}^{4} a_{i}^{k}\left(\rho_{0}^{2} J^{-2}\right),{ }_{k} \bar{\partial}^{4} v^{i} d x, \\
& \mathcal{I}_{2}=\int_{\Omega} a_{i}^{k}\left(\rho_{0}^{2} \bar{\partial}^{4} J^{-2}\right),{ }_{k} \bar{\partial}^{4} v^{i} d x, \\
& \mathcal{I}_{3}=\int_{\Omega} a_{i}^{k}\left(\bar{\partial}^{4} \rho_{0}^{2} J^{-2}\right), \bar{\partial}^{4} v^{i} d x, \\
& \mathcal{R}=\sum_{l=1}^{3} c_{l} \int_{\Omega} \bar{\partial}^{4-l} a_{i}^{k} \bar{\partial}^{l}\left(\rho_{0}^{2} J^{-2}\right),{ }_{k} \bar{\partial}^{4} v^{i} d x .
\end{aligned}
$$

The last integral introduces our notation $\mathcal{R}$ for the remainder, which throughout the paper will consist of integrals of lower-order terms which can, via elementary inequalities together with our assumptions (6.7), easily be shown to satisfy the following estimate:

$$
\int_{0}^{T} \mathcal{R}(t) d t \leq M_{0}+\delta \sup _{t \in[0, T]} E(t)+C T P\left(\sup _{t \in[0, T]} E(t)\right) .
$$

The sum of $\int_{0}^{T}\left[\mathcal{I}_{1}(t)+\mathcal{I}_{2}(t)+\mathcal{I}_{3}(t)\right] d t$ together with the estimates for curl $\eta$ given by Proposition 6.1 will provide the remaining energy contribution $\int_{\Omega} \rho_{0}^{2}(x, t)\left|\bar{\partial}^{4} D \eta\right|^{2} d x$ plus error terms which have the same bound as $\mathcal{R}$. 
Analysis of $\int_{0}^{T} \mathcal{R} d t$. Using the identity (4.5), we integrate by parts with respect to $x_{k}$ and then with respect to the time derivative $\partial_{t}$, and use (4.5) to obtain that

$$
\begin{aligned}
\mathcal{R}= & -\sum_{l=1}^{3} c_{l} \int_{0}^{T} \int_{\Omega} \bar{\partial}^{4-l} a_{i}^{k} \bar{\partial}^{l}\left(\rho_{0}^{2} J^{-2}\right) \bar{\partial}^{4} v^{i},{ }_{k} d x d t \\
= & \sum_{l=1}^{3} c_{l} \int_{0}^{T} \int_{\Omega}\left(\bar{\partial}^{4-l} a_{i}^{k} \bar{\partial}^{l}\left(\rho_{0}^{2} J^{-2}\right)\right)_{t} \bar{\partial}^{4} \eta^{i},{ }_{k} d x d t \\
& -\sum_{l=1}^{3} c_{l} \int_{\Omega} \bar{\partial}^{4-l} a_{i}^{k} \bar{\partial}^{l}\left(\rho_{0}^{2} J^{-2}\right) \bar{\partial}^{4} \eta^{i},\left.{ }_{k} d x\right|_{0} ^{T} .
\end{aligned}
$$

Notice that when $l=3$, the highest-order integrand in the spacetime integral on the right-hand side scales like $\ell\left[\bar{\partial} D \eta \rho_{0} \bar{\partial}^{3} \partial_{t} J^{-2}+\bar{\partial} D v \rho_{0} \bar{\partial}^{3} J^{-2}\right] \rho_{0} \bar{\partial}^{4} D \eta$, where $\ell$ denotes an $L^{\infty}((0, T) \times \Omega)$ function. Since $\left\|\rho_{0} \partial_{t}^{2} J^{-2}(t)\right\|_{3}^{2}$ is contained in the energy function $E(t)$ and since $\bar{\partial} D \eta(t) \in L^{\infty}(\Omega)$, the first summand is estimated using an $L^{\infty}$. $L^{2}-L^{2}$ Hölder's inequality, while for the second summand, we use that $\left\|\rho_{0} J^{-2}(t)\right\|_{4}^{2}$ is contained in $E(t)$ together with an $L^{4}-L^{4}-L^{2}$ Hölder's inequality.

When $l=1$, the integrand in the spacetime integral on the right-hand side scales like $\ell\left[\bar{\partial} D \eta \rho_{0} \bar{\partial}^{3} a_{t}{ }_{i}+\bar{\partial} D v \rho_{0} \bar{\partial}^{3} a_{i}^{k}\right] \rho_{0} \bar{\partial}^{4} \eta^{i},{ }_{k}$. Since $\left\|\rho_{0} \bar{\partial}^{3} D v_{t}(t)\right\|_{0}^{2}$ is contained in the energy function $E(t)$ and since $\bar{\partial} D \eta \in L^{\infty}(\Omega)$, the first summand is estimated using an $L^{\infty}-L^{2}-L^{2}$ Hölder's inequality. We write the second summand as

$$
\bar{\partial} D v \rho_{0} \bar{\partial}^{3} a_{i}^{\beta} \rho_{0} \bar{\partial}^{4} \eta^{i}, \beta+\bar{\partial} D v \rho_{0} \bar{\partial}^{3} a_{i}^{3} \rho_{0} \bar{\partial}^{4} \eta^{i}, 3 \text {. }
$$

We estimate

$$
\begin{aligned}
\int_{0}^{T} & \int_{\Omega} \bar{\partial} D v \rho_{0} \bar{\partial}^{3} a_{i}^{\beta} \rho_{0} \bar{\partial}^{4} \eta^{i}, \beta d x d t \\
= & -\int_{0}^{T} \int_{\Omega}\left[\bar{\partial} D v \rho_{0} \bar{\partial}^{3} a_{i}^{\beta}, \beta \rho_{0} \bar{\partial}^{4} \eta^{i}+\bar{\partial} D v,_{\beta} \rho_{0} \bar{\partial}^{3} a_{i}^{\beta} \rho_{0} \bar{\partial}^{4} \eta^{i}\right] d x d t \\
\leq & C \int_{0}^{T}\left(\|\bar{\partial} D v(t)\|_{L^{3}(\Omega)}\left\|\rho_{0} \bar{\partial}^{4} a(t)\right\|_{0}\left\|\rho_{0} \bar{\partial}^{4} \eta(t)\right\|_{L^{6}(\Omega)}\right. \\
& \left.+\left\|\bar{\partial}^{2} D v(t)\right\|_{L^{3}(\Omega)}\left\|\rho_{0} \bar{\partial}^{4} \eta(t)\right\|_{L^{6}(\Omega)}\left\|\bar{\partial}^{3} a\right\|_{0}\right) d t \\
\leq & C \int_{0}^{T}\left(\|\bar{\partial} D v(t)\|_{H^{0.5}(\Omega)}\left\|\rho_{0} \bar{\partial}^{4} a(t)\right\|_{0}\left\|\rho_{0} \bar{\partial}^{4} \eta(t)\right\|_{1}\right. \\
& \left.+\left\|\bar{\partial}^{2} D v(t)\right\|_{H^{0.5}(\Omega)}\left\|\rho_{0} \bar{\partial}^{4} \eta(t)\right\|_{1}\left\|\bar{\partial}^{3} a\right\|_{0}\right) d t \\
\leq & C \int_{0}^{T}\left(\|v(t)\|_{H^{3.5}(\Omega)}\left\|\rho_{0} \bar{\partial}^{4} D \eta(t)\right\|_{0}^{2}+\|v(t)\|_{H^{2.5}(\Omega)}\left\|\rho_{0} \bar{\partial}^{4} D \eta(t)\right\|_{0}\|\eta(t)\|_{4}\right. \\
& \left.+\|v(t)\|_{H^{3.5}(\Omega)}\|\eta(t)\|_{4}^{2}\right) d t,
\end{aligned}
$$

where we have used Hölder's inequality, followed by the Sobolev embeddings

$$
H^{0.5}(\Omega) \hookrightarrow L^{3}(\Omega) \text { and } H^{1}(\Omega) \hookrightarrow L^{6}(\Omega) .
$$


We also rely on the interpolation estimate

$$
\begin{aligned}
\|v\|_{L^{2}\left(0, T ; H^{3.5}(\Omega)\right)}^{2} & \leq\left. C\left(\|v(t)\|_{3}\|\eta\|_{4}\right)\right|_{T} ^{0}+C\left\|v_{t}\right\|_{L^{2}\left(0, T ; H^{3}(\Omega)\right)}\|\eta\|_{L^{2}\left(0, T ; H^{4}(\Omega)\right)} \\
& \leq M_{0}+\delta \sup _{t \in[0, T]}\|\eta(t)\|_{4}^{2}+C T \sup _{t \in[0, T]}\left(\|\eta(t)\|_{4}^{2}+\left\|v_{t}(t)\right\|_{3}^{2}\right),
\end{aligned}
$$

where the last inequality follows from Young's and Jensen's inequalities. Using this together with the Cauchy-Schwarz inequality, (6.11) is bounded by $C T P\left(\sup _{t \in[0, T]}\right.$ $E(t))$. Next, since (4.6) shows that each component of $a_{i}^{3}$ is quadratic in $\bar{\partial} \eta$, we see that the same analysis shows the spacetime integral of $\bar{\partial} D v \rho_{0} \bar{\partial}^{3} a_{i}^{3} \rho_{0} \bar{\partial}^{4} \eta^{i}, 3$ has the same bound, and so we have estimated the case $l=1$.

For the case that $l=2$, the integrand in the spacetime integral on the right-hand side of the expression for $\mathcal{R}$ scales like $\ell \bar{\partial}^{2} D \eta \bar{\partial}^{2} D v \rho_{0} \bar{\partial}^{4} D \eta$, so that an $L^{6}-L^{3}-L^{2}$ Hölder's inequality, followed by the same analysis as for the case $l=1$ provides the same bound as for the case $l=1$.

To deal with the space integral on the right-hand side of the expression for $\mathcal{R}$, the integral at time $t=0$ is equal to zero since $\eta(x, 0)=x$, whereas the integral evaluated at $t=T$ is written, using the fundamental theorem of calculus, as

$$
\begin{aligned}
& -\sum_{l=1}^{3} c_{l} \int_{\Omega} \rho_{0} \bar{\partial}^{4-l} a_{i}^{k} \bar{\partial}^{l} J^{-2} \rho_{0} \bar{\partial}^{4} \eta^{i},\left.{ }_{k} d x\right|_{t=T} \\
& =-\sum_{l=1}^{3} c_{l} \int_{\Omega} \rho_{0} \int_{0}^{T}\left(\bar{\partial}^{4-l} a_{i}^{k} \bar{\partial}^{l} J^{-2}\right)_{t} \rho_{0} \bar{\partial}^{4} \eta^{i},_{k}(T) d x,
\end{aligned}
$$

which can be estimated in the identical fashion as the corresponding spacetime integral. As such, we have shown that $\mathcal{R}$ has the claimed bound (6.10).

Analysis of the integral $\mathcal{I}_{1}$. Because $\rho_{0}=0$ on $\Gamma=\left\{x_{3}=1\right\}$, we use the identity (4.5) to integrate by parts with respect to $x_{k}$ to find that

$$
\begin{aligned}
\mathcal{I}_{1} & =-\int_{\Omega} \rho_{0}^{2} J^{-2} \bar{\partial}^{4} a_{i}^{k} \bar{\partial}^{4} v^{i},{ }_{k} d x+\int_{\left\{x_{3}=0\right\}} \rho_{0}^{2} J^{-2} \bar{\partial}^{4} a_{i}^{3} \bar{\partial}^{4} v^{i} d x_{1} d x_{2} \\
& =-\int_{\Omega} \rho_{0}^{2} J^{-2} \bar{\partial}^{4} a_{i}^{k} \bar{\partial}^{4} v^{i},{ }_{k} d x,
\end{aligned}
$$

since on the fixed boundary $\left\{x_{3}=0\right\}, \eta^{3}=x_{3}$ so that according to (4.6), the components $a_{1}^{3}=0$ and $a_{2}^{3}=0$ on $\left\{x_{3}=0\right\}$, and $v^{3}=0$ on $\left\{x_{3}=0\right\}$, so that $\bar{\partial}^{4} a_{i}^{3} \bar{\partial}^{4} v^{i}=0$ on $\left\{x_{3}=0\right\}$.

To estimate $\mathcal{I}_{1}$, we use the formula (4.3) for horizontally differentiating the cofactor matrix:

$$
\mathcal{I}_{1}=\int_{\Omega} \rho_{0}^{2} J^{-3} \bar{\partial}^{4} \eta^{r},_{s}\left[a_{i}^{s} a_{r}^{k}-a_{r}^{s} a_{i}^{k}\right] \bar{\partial}^{4} v^{i},{ }_{k} d x+\mathcal{R}
$$

where the remainder $\mathcal{R}$ satisfies (6.10). We decompose the highest-order term in $\mathcal{I}_{1}$ as the sum of the following two integrals: 


$$
\begin{aligned}
& \mathcal{I}_{1 a}=\int_{\Omega} \rho_{0}{ }^{2} J^{-3}\left(\bar{\partial}^{4} \eta^{r},{ }_{s} a_{i}^{s}\right)\left(\bar{\partial}^{4} v^{i},{ }_{k} a_{r}^{k}\right) d x, \\
& \mathcal{I}_{1 b}=-\int_{\Omega} \rho_{0}{ }^{2} J^{-3}\left(\bar{\partial}^{4} \eta^{r},{ }_{s} a_{r}^{s}\right)\left(\bar{\partial}^{4} v^{i},{ }_{k} a_{i}^{k}\right) d x .
\end{aligned}
$$

Since $v=\eta_{t}, \mathcal{I}_{1 a}$ is an exact derivative modulo an antisymmetric commutation with respect to the free indices $i$ and $r$; namely,

$$
\bar{\partial}^{4} \eta^{r},_{s} a_{i}^{s} \bar{\partial}^{4} v^{i}{ }_{k} a_{r}^{k}=\bar{\partial}^{4} \eta^{i}{ }_{s} a_{r}^{s} \bar{\partial}^{4} v^{i}{ }_{k} a_{r}^{k}+\left(\bar{\partial}^{4} \eta^{r},_{s} a_{i}^{s}-\bar{\partial}^{4} \eta^{i},_{s} a_{r}^{s}\right) \bar{\partial}^{4} v^{i},_{k} a_{r}^{k} .
$$

Using the notation

$$
\begin{aligned}
{\left[D_{\eta} F\right]_{r}^{i} } & =a_{r}^{s} F^{i}, \quad \text { for any vector field } F, \\
\bar{\partial}^{4} \eta^{i}{ }_{s} a_{r}^{s} \bar{\partial}^{4} v^{i}{ }_{k} a_{r}^{k} & =\frac{1}{2} \frac{d}{d t}\left|D_{\eta} \bar{\partial}^{4} \eta\right|^{2}-\frac{1}{2} \bar{\partial}^{4} \eta^{r},_{s} \bar{\partial}^{4} \eta^{i},_{k} \quad\left(a_{r}^{s} a_{i}^{k}\right)_{t},
\end{aligned}
$$

so the first term on the right-hand side of (6.13) produces an exact derivative in time.

For the second term on the right-hand side of (6.13), note the identity

$$
\left(\bar{\partial}^{4} \eta^{r}{ }_{,} a_{i}^{s}-\bar{\partial}^{4} \eta^{i}{ }_{,} a_{r}^{s}\right) \bar{\partial}^{4} v^{i}{ }_{k} a_{r}^{k}=-J^{2} \varepsilon_{i j k} \bar{\partial}^{4} \eta^{k}{ }_{r} A_{j}^{r} \varepsilon_{i m n} \bar{\partial}^{4} v^{n}{ }_{s} A_{m}^{s} .
$$

We have used the permutation symbol $\varepsilon$ to encode the anti-symmetry in this relation, and the basic fact that the trace of the product of symmetric and antisymmetric matrices is equal to zero.

Recalling our notation $\left[\operatorname{curl}_{\eta} F\right]^{i}=\varepsilon_{i j k} F^{k},{ }_{r} A_{j}^{r}$, (6.15) can be written as

$$
\left(\bar{\partial}^{4} \eta^{r},{ }_{s} a_{i}^{s}-\bar{\partial}^{4} \eta^{i}{ }_{s} a_{r}^{s}\right) \bar{\partial}^{4} v^{i},{ }_{k} a_{r}^{k}=-J^{2} \operatorname{curl}_{\eta} \bar{\partial}^{4} \eta \cdot \operatorname{curl}_{\eta} \bar{\partial}^{4} v
$$

which can also be written as an exact derivative in time:

$$
\begin{aligned}
\operatorname{curl}_{\eta} \bar{\partial}^{4} \eta \cdot \operatorname{curl}_{\eta} \bar{\partial}^{4} v= & \frac{1}{2} \frac{d}{d t}\left|\operatorname{curl}_{\eta} \bar{\partial}^{4} \eta\right|^{2}-\bar{\partial}^{4} \eta^{k},{ }_{r} \bar{\partial}^{4} \eta^{k},_{s}\left(A_{j}^{r} A_{j}^{s}\right)_{t} \\
& +\bar{\partial}^{4} \eta^{k},_{r} \bar{\partial}^{4} \eta^{j},_{s}\left(A_{j}^{r} A_{k}^{s}\right)_{t} .
\end{aligned}
$$

The terms in (6.14) and (6.17) which are not the exact time derivatives are quadratic in $\rho_{0} \bar{\partial}^{4} D \eta$ with coefficients in $L^{\infty}([0, T] \times \Omega)$; denoting the integral over $\Omega$ of such terms by $\mathcal{Q}_{\rho_{0} \bar{\partial}^{4} D \eta}$,

$\mathcal{I}_{1 a}=\frac{1}{2} \frac{d}{d t} \int_{\Omega} \rho_{0}{ }^{2} J^{-3}\left|D_{\eta} \bar{\partial}^{4} \eta\right|^{2} d x-\frac{1}{2} \frac{d}{d t} \int_{\Omega} \rho_{0}^{2} J^{-1}\left|\operatorname{curl}_{\eta} \bar{\partial}^{4} \eta\right|^{2} d x+\mathcal{Q}_{\rho_{0} \bar{\partial}^{4} D \eta}+\mathcal{R}$,

where $\int_{0}^{T}\left|\mathcal{Q}_{\rho_{0} \bar{\partial}^{4} D \eta}\right| d t \leq C T P\left(\sup _{t \in[0, T]} E(t)\right)$, and $\mathcal{R}$ satisfies (6.10).

With the notation $\operatorname{div}_{\eta} F=A_{i}^{j} F^{i},{ }_{j}$, the differentiation formula (4.1) shows that $\mathcal{I}_{1 b}$ can be written as

$$
\mathcal{I}_{1 b}=-\frac{1}{2} \frac{d}{d t} \int_{\Omega} \rho_{0}^{2} J^{-1}\left|\operatorname{div}_{\eta} \bar{\partial}^{4} \eta\right|^{2} d x+\mathcal{Q}_{\rho_{0} \bar{\partial}^{4} D \eta}+\mathcal{R} .
$$

It follows that

$$
\begin{aligned}
\mathcal{I}_{1} & =\frac{1}{2} \frac{d}{d t} \int_{\Omega} \rho_{0}^{2}\left(J^{-3}\left|D_{\eta} \bar{\partial}^{4} \eta\right|^{2}-J^{-1}\left|\operatorname{curl}_{\eta} \bar{\partial}^{4} \eta\right|^{2}-J^{-1}\left|\operatorname{div}_{\eta} \bar{\partial}^{4} \eta\right|^{2}\right) d x+\mathcal{R} \\
& =\frac{1}{2} \frac{d}{d t} \int_{\Omega} \rho_{0}^{2}\left(\left|D \bar{\partial}^{4} \eta\right|^{2}-J^{-1}\left|\operatorname{curl}_{\eta} \bar{\partial}^{4} \eta\right|^{2}-J^{-1}\left|\operatorname{div}_{\eta} \bar{\partial}^{4} \eta\right|^{2}\right) d x+\mathcal{R},
\end{aligned}
$$


where we have used the fundamental theorem of calculus for the second equality on the term $J^{-3} D_{\eta} \bar{\partial}^{4} \eta$. Since $\frac{1}{2}<J(t)<\frac{3}{2}$, we see that

$$
\begin{aligned}
\int_{0}^{T} \mathcal{I}_{1}(t) d t= & \frac{1}{2} \int_{\Omega} \rho_{0}^{2}\left(\left|D \bar{\partial}^{4} \eta(T)\right|^{2}-J^{-1}\left|\operatorname{curl}_{\eta} \bar{\partial}^{4} \eta(T)\right|^{2}-J^{-1}\left|\operatorname{div}_{\eta} \bar{\partial}^{4} \eta\right|^{2}(T)\right) d x \\
& -M_{0}+\int_{0}^{T} \mathcal{R}(t) d t
\end{aligned}
$$

Analysis of the integral $\mathcal{I}_{2}$. Integration by parts once again, using (4.5), yields

$$
\mathcal{I}_{2}=-\int_{\Omega} \rho_{0}^{2} \bar{\partial}^{4} J^{-2} a_{i}^{k} \bar{\partial}^{4} v^{i}, k d x .
$$

Since $\bar{\partial}^{4} J^{-2}=-2 J^{-3} \bar{\partial}^{4} J$ plus lower-order terms, which have at most three horizontal derivatives acting on $J$. For such lower-order terms, we integrate by parts with respect to $\partial_{t}$, and estimate the resulting integrals in the same manner as we estimated the remainder term $\mathcal{R}$, and obtain the same bound.

Thus,

$$
\begin{aligned}
\mathcal{I}_{2} & =2 \int_{\Omega} \rho_{0}^{2} J^{-3} a_{s}^{r} \bar{\partial}^{4} \eta^{s},{ }_{r} \quad a_{i}^{k} \bar{\partial}^{4} v^{i},{ }_{k} d x+\mathcal{R} \\
& =\frac{d}{d t} \int_{\Omega} \rho_{0}^{2} J^{-3} a_{s}^{r} \bar{\partial}^{4} \eta^{s},{ }_{r} \quad a_{i}^{k} \bar{\partial}^{4} \eta^{i}{ }_{k} d x-\int_{\Omega} \rho_{0}^{2}\left(J^{-3} a_{s}^{r} a_{i}^{k}\right)_{t} \bar{\partial}^{4} \eta^{s},{ }_{r} \bar{\partial}^{4} \eta^{i},{ }_{k} d x+\mathcal{R} .
\end{aligned}
$$

Given our identities for differentiating $a$ and $J$, the Sobolev embedding theorem together with our assumptions (6.7) and the Cauchy-Schwarz inequality show that

$$
\int_{0}^{T} \int_{\Omega} \rho_{0}^{2}\left(J^{-3} a_{s}^{r} a_{i}^{k}\right)_{t} \bar{\partial}^{4} \eta^{s}, r \bar{\partial}^{4} \eta^{i},{ }_{k} d x d t \leq C T \sup _{t \in[0, T]} E(t) ;
$$

consequently, we can write

$$
\int_{\Omega} \rho_{0}^{2} J^{-3} a_{s}^{r} \bar{\partial}^{4} \eta^{s}, r \quad a_{i}^{k} \bar{\partial}^{4} \eta^{i}, k d x-M_{0}=\int_{0}^{t}\left[\mathcal{I}_{2}\left(t^{\prime}\right)+\mathcal{R}\left(t^{\prime}\right)\right] d t^{\prime} .
$$

On the other hand,

$$
\begin{aligned}
\int_{\Omega} & \rho_{0}^{2} J^{-3} a_{s}^{r} \bar{\partial}^{4} \eta^{s}, r \\
= & \int_{i}^{k} \bar{\partial}^{4} \eta^{i},{ }_{k} d x \\
& \rho_{0}^{2} J^{-3}\left(\bar{\partial}^{4} \operatorname{div} \eta+\bar{\partial}^{4} \eta^{s}, r \int_{0}^{t} a_{t_{s}}^{r} d t^{\prime}\right)\left(\bar{\partial}^{4} \operatorname{div} \eta+\bar{\partial}^{4} \eta^{i},{ }_{k} \int_{0}^{t} a_{t_{i}}^{k} d t^{\prime}\right) d x \\
= & \int_{\Omega} \rho_{0}^{2} J^{-3}\left|\bar{\partial}^{4} \operatorname{div} \eta\right|^{2} d x+2 \int_{\Omega} \rho_{0}^{2} J^{-2} \bar{\partial}^{4} \operatorname{div} \eta \bar{\partial}^{4} \eta^{s}, r \int_{0}^{t} a_{t s}^{r} d t^{\prime} d x \\
& +\int_{\Omega} \rho_{0}^{2} J^{-3} \bar{\partial}^{4} \eta^{s}, r \int_{0}^{t} a_{t_{s}}^{r} d t^{\prime} \bar{\partial}^{4} \eta^{i}, k \int_{0}^{t} a_{t i}^{k} d t^{\prime} d x
\end{aligned}
$$

Yet another application of the Sobolev embedding theorem together with our assumptions (6.7) and the Cauchy-Schwarz inequality shows that the second and third integrals 
on the right-hand side are bounded by $M_{0}+C T \sup _{t \in[0, T]} E(t)$, so that combining (6.19) and (6.20), we find that

$$
\int_{0}^{T} \mathcal{I}_{2}(t) d t=\int_{\Omega} \rho_{0}^{2} J^{-1}\left|\bar{\partial}^{4} \operatorname{div} \eta\right|^{2} d x-M_{0}+\int_{0}^{T} \mathcal{R}(t) d t .
$$

Analysis of the integral $\mathcal{I}_{3}$. Integration by parts using (4.5) shows that

$$
\begin{aligned}
\int_{0}^{T} \mathcal{I}_{3}(t) d t= & -\int_{0}^{T} \int_{\Omega} \bar{\partial}^{4} \rho_{0}^{2} J^{-2} a_{i}^{k} \bar{\partial}^{4} v^{i},_{k} d x d t \\
= & \int_{0}^{T} \int_{\Omega} \bar{\partial}^{4} \rho_{0}^{2}\left(J^{-2} a_{i}^{k}\right)_{t} \bar{\partial}^{4} \eta^{i},{ }_{k} d x d t-\int_{\Omega} \bar{\partial}^{4} \rho_{0}^{2} J^{-2} a_{i}^{k} \bar{\partial}^{4} \eta^{i},\left.{ }_{k} d x\right|_{t=T} \\
= & \int_{0}^{T} \int_{\Omega} \bar{\partial}^{4} \rho_{0}^{2}\left(J^{-2} a_{i}^{k}\right)_{t} \bar{\partial}^{4} \eta^{i},_{k} d x d t-\int_{\Omega} \bar{\partial}^{4} \rho_{0}^{2} \bar{\partial}^{4} \operatorname{div} \eta(T) d x \\
& -\int_{\Omega} \bar{\partial}^{4} \rho_{0}^{2} \int_{0}^{T} J^{-2} a_{i}^{k} d t \bar{\partial}^{4} \eta^{i},{ }_{k}(T) d x,
\end{aligned}
$$

so that by the Cauchy-Schwarz inequality and Young's inequality,

$$
\int_{0}^{T} \mathcal{I}_{3}(t) d t \leq M_{0}+\delta \sup _{t \in[0, T]} E(t)+C T P\left(\sup _{t \in[0, T]} E(t)\right) .
$$

Summing inequalities. We integrate (6.9) from 0 to $T$, and sum (6.10), (6.18), (6.21), and (6.22) to find that

$$
\begin{aligned}
& \sup _{t \in[0, T]} \frac{1}{2}\left[\int_{\Omega} \rho_{0}^{2}\left|\bar{\partial}^{4} D \eta\right|^{2} d x+\int_{\Omega} \rho_{0}^{2} J^{-1}\left|\bar{\partial}^{4} \operatorname{div} \eta\right|^{2} d x-\int_{\Omega} \rho_{0}^{2} J^{-1}\left|\bar{\partial}^{4} \operatorname{curl} \eta\right|^{2} d x\right] \\
& \quad \leq M_{0}+\delta \sup _{t \in[0, T]} E(t)+C T P\left(\sup _{t \in[0, T]} E(t)\right) .
\end{aligned}
$$

Note the factor of $\frac{1}{2}$ in (6.18) which, in conjunction with (6.21), gives the positivity of the divergence term.

Adding to this the inequality (6.1), and possibly readjusting our constants, we obtain the desired result, and complete the proof of the proposition.

With the unit tangent vectors $T_{1}=(1,0,0)$ and $T_{2}=(0,1,0), \eta \cdot T_{\alpha}=\eta^{\alpha}$ for $\alpha=1,2$, and we have the following

Corollary 6.3. For $\alpha=1,2$,

$$
\sup _{t \in[0, T]}\left|\eta^{\alpha}\right|_{3.5}^{2} \leq M_{0}+C T P\left(\sup _{t \in[0, T]} E(t)\right) .
$$

Proof. The weighted embedding estimate (2.1) shows that

$$
\left\|\bar{\partial}^{4} \eta\right\|_{0}^{2} \leq C \int_{\Omega} \rho_{0}^{2}\left(\left|\bar{\partial}^{4} \eta\right|^{2}+\left|\bar{\partial}^{4} D \eta\right|^{2}\right) d x .
$$

Now

$$
\sup _{t \in[0, T]} \int_{\Omega} \rho_{0}^{2}\left|\bar{\partial}^{4} \eta\right|^{2} d x=\sup _{t \in[0, T]} \int_{\Omega} \rho_{0}^{2}\left|\int_{0}^{t} \bar{\partial}^{4} v d t^{\prime}\right|^{2} d x \leq T^{2} \sup _{t \in[0, T]}\left\|\sqrt{\rho_{0}} \bar{\partial}^{4} v\right\|_{0}^{2} .
$$


It follows from Proposition 6.2 that

$$
\sup _{t \in[0, T]}\left\|\bar{\partial}^{4} \eta\right\|_{0}^{2} \leq M_{0}+C T P\left(\sup _{t \in[0, T]} E(t)\right) .
$$

According to our curl estimates (6.1), $\sup _{t \in[0, T]}\|\operatorname{curl} \eta\|_{3}^{2} \leq M_{0}+C T P\left(\sup _{t \in[0, T]} E(t)\right)$, from which it follows that

$$
\sup _{t \in[0, T]}\left\|\bar{\partial}^{4} \operatorname{curl} \eta\right\|_{H^{1}(\Omega)^{\prime}}^{2} \leq M_{0}+C T P\left(\sup _{t \in[0, T]} E(t)\right),
$$

since $\bar{\partial}$ is a horizontal derivative, and integration by parts with respect to $\bar{\partial}$ does not produce any boundary contributions. From the tangential trace inequality (5.2), we find that

$$
\sup _{t \in[0, T]}\left|\bar{\partial}^{4} \eta^{\alpha}\right|_{-1 / 2}^{2} \leq M_{0}+C T P\left(\sup _{t \in[0, T]} E(t)\right),
$$

from which the assertion of the corollary follows.

\subsection{The $\partial_{t}^{8}$-problem.}

Proposition 6.4. For $\delta>0$ and letting the constant $M_{0}$ depend on $1 / \delta$,

$$
\begin{aligned}
& \sup _{t \in[0, T]}\left(\int_{\Omega} \rho_{0}\left|\partial_{t}^{8} v(x, t)\right|^{2} d x+\int_{\Omega} \rho_{0}^{2}(x, t)\left|\partial_{t}^{7} D v(x, t)\right|^{2} d x\right) \\
& \quad \leq M_{0}+\delta \sup _{t \in[0, T]} E(t)+C T P\left(\sup _{t \in[0, T]} E(t)\right) .
\end{aligned}
$$

Proof. Letting $\partial_{t}^{8}$ act on $\rho_{0} v_{t}^{i}+a_{i}^{k}\left(\rho_{0}^{2} J^{-2}\right), k=0$, and taking the $L^{2}(\Omega)$-inner product with $\partial_{t}^{8} v^{i}$, we obtain

$$
\begin{aligned}
& \frac{1}{2} \frac{d}{d t} \int_{\Omega} \rho_{0}\left|\partial_{t}^{8} v\right|^{2} d x+\int_{\Omega} \partial_{t}^{8} a_{i}^{k}\left(\rho_{0}^{2} J^{-2}\right),{ }_{k} \partial_{t}^{8} v^{i} d x+\int_{\Omega} a_{i}^{k}\left(\rho_{0}^{2} \partial_{t}^{8} J^{-2}\right),{ }_{k} \partial_{t}^{8} v^{i} d x \\
& \quad=\sum_{l=1}^{7} c_{l} \int_{\Omega} \partial_{t}^{8-l} a_{i}^{k}\left(\rho_{0}^{2} \partial_{t}^{l} J^{-2}\right),{ }_{k} d x
\end{aligned}
$$

Integrating the first term from 0 to $t \in(0, T]$ produces the first term on the left-hand side of (6.23).

We define the following three integrals:

$$
\begin{aligned}
\mathcal{I}_{1} & =\int_{\Omega} \partial_{t}^{8} a_{i}^{k}\left(\rho_{0}^{2} J^{-2}\right),{ }_{k} \partial_{t}^{8} v^{i} d x, \\
\mathcal{I}_{2} & =\int_{\Omega} a_{i}^{k}\left(\rho_{0}^{2} \partial_{t}^{8} J^{-2}\right),{ }_{k} \partial_{t}^{8} v^{i} d x, \\
\mathcal{R} & =\sum_{l=1}^{7} c_{l} \int_{\Omega} \partial_{t}^{8-l} a_{i}^{k}\left(\rho_{0}^{2} \partial_{t}^{l} J^{-2}\right),{ }_{k} \partial_{t}^{8} v^{i} d x .
\end{aligned}
$$

The sum of $\int_{0}^{T}\left[\mathcal{I}_{1}(t)+\mathcal{I}_{2}(t)\right] d t$ together with the curl estimates given by Proposition 6.1 will provide the remaining energy contribution $\int_{\Omega} \rho_{0}^{2}(x, t)\left|\partial_{t}^{7} D v\right|^{2} d x$ plus error terms which have the same bound as $\mathcal{R}$, namely (6.10). 
Analysis of $\int_{0}^{T} \mathcal{R} d t$. We use the identity (4.5) to integrate by parts with respect to $x_{k}$ and then with respect to the time derivative $\partial_{t}$ to obtain that

$$
\begin{aligned}
\mathcal{R}= & -\sum_{l=1}^{7} c_{l} \int_{0}^{T} \int_{\Omega} \partial_{t}^{8-l} a_{i}^{k} \rho_{0}^{2} \partial_{t}^{l} J^{-2} \partial_{t}^{8} v^{i},{ }_{k} d x d t \\
= & \sum_{l=1}^{7} c_{l} \int_{0}^{T} \int_{\Omega} \rho_{0}\left(\partial_{t}^{8-l} a_{i}^{k} \partial_{t}^{l} J^{-2}\right)_{t} \rho_{0} \partial_{t}^{7} v^{i},{ }_{k} d x d t \\
& -\sum_{l=1}^{7} c_{l} \int_{\Omega} \rho_{0} \partial_{t}^{8-l} a_{i}^{k} \bar{\partial}^{l} J^{-2} \rho_{0} \partial_{t}^{7} v^{i},\left.{ }_{k} d x\right|_{0} ^{T} .
\end{aligned}
$$

Notice that when $l=7$, the integrand in the spacetime integral on the right-hand side scales like $\ell\left[D v_{t} \rho_{0} \partial_{t}^{6} D v+D v \rho_{0} \partial_{t}^{7} D v\right] \rho_{0} \partial_{t}^{7} D v$, where $\ell$ denotes an $L^{\infty}(\Omega)$ function. Since $\left\|\rho_{0} \partial_{t}^{7} D v(t)\right\|_{0}^{2}$ is contained in the energy function $E(t), D v_{t}(t)$ is bounded in $L^{\infty}(\Omega)$, and since we can write $\rho_{0} \partial_{t}^{6} D v(t)=\rho_{0} \partial_{t}^{6} D v(0)+\int_{0}^{t} \rho_{0} \partial_{t}^{7} D v\left(t^{\prime}\right) d t^{\prime}$, the first and second summands are both estimated using an $L^{\infty}-L^{2}-L^{2}$ Hölder's inequality.

The case $l=6$ is estimated exactly the same way as the case $l=3$ in the proof of Proposition 6.2. For the case $l=5$, the integrand in the spacetime integral scales like $\ell\left[D v_{t t} \rho_{0} \partial_{t}^{6} J^{-2}+D v_{t t t} \rho_{0} D v_{t t t t}\right] \rho_{0} \partial_{t}^{7} D v$. Both summands can be estimated using an $L^{3}-L^{6}-L^{2}$ Hölder's inequality. The case $l=4$ is treated as the case $l=5$. The case $l=3$ is also treated in the same way as $l=5$. The case $l=2$ is estimated exactly the same way as the case $l=1$ in the proof of Proposition 6.2. The case $l=1$ is treated in the same way as the case $l=7$.

To deal with the space integral on the right-hand side of the expression for $\mathcal{R}$, the integral at time $t=0$ is bounded by $M_{0}$, whereas the integral evaluated at $t=T$ is written, using the fundamental theorem of calculus, as

$$
\begin{aligned}
\sum_{l=1}^{7} c_{l} \int_{\Omega} \rho_{0} \partial_{t}^{8-l} a_{i}^{k} \partial_{t}^{l} J^{-2} \rho_{0} \partial_{t}^{7} v^{i},\left.{ }_{k} d x\right|_{t=T} \\
=\sum_{l=1}^{7} c_{l} \int_{\Omega} \rho_{0} \partial_{t}^{8-l} a_{i}^{k}(0) \partial_{t}^{l} J^{-2}(0) \rho_{0} \partial_{t}^{7} v^{i},{ }_{k}(T) d x \\
\quad+\sum_{l=1}^{7} c_{l} \int_{\Omega} \rho_{0} \int_{0}^{T}\left(\partial_{t}^{8-l} a_{i}^{k} \partial_{t}^{l} J^{-2}\right)_{t} d t^{\prime} \rho_{0} \partial_{t}^{7} v^{i},{ }_{k}(T) d x .
\end{aligned}
$$

The first integral on the right-hand side is estimated using Young's inequality, and is bounded by $M_{0}+\delta \sup _{t \in[0, T]} E(t)$, while the second integral can be estimated in the identical fashion as the corresponding spacetime integral. As such, we have shown that $\mathcal{R}$ has the claimed bound (6.10).

Analysis of the integral $\mathcal{I}_{1}$. As to the term $\mathcal{I}_{1}$, using the identity (4.4), the same computation as for the $\bar{\partial}^{4}$-differentiated problem shows that

$$
\begin{aligned}
\rho_{0}^{2}\left(\partial_{t}^{7} v^{r},{ }_{s} A_{i}^{s}\right)\left(\partial_{t}^{8} v^{i},{ }_{k} A_{r}^{k}\right)= & \frac{1}{2} \frac{d}{d t}\left|\rho_{0} D_{\eta} \partial_{t}^{7} v(t)\right|^{2}-\frac{1}{2} \frac{d}{d t}\left|\rho_{0} \operatorname{curl}_{\eta} \partial_{t}^{7} v(t)\right|^{2} \\
& +\frac{1}{2} \rho_{0}{ }^{2} \partial_{t}^{7} v^{k},{ }_{r} \partial_{t}^{7} v^{b},_{s}\left(A_{j}^{r} A_{m}^{s}\right)_{t}\left[\delta_{m}^{j} \delta_{b}^{k}-\delta_{b}^{j} \delta_{m}^{k}\right],
\end{aligned}
$$


and

$$
-\rho_{0}^{2}\left(\partial_{t}^{7} v^{r},{ }_{s} A_{r}^{s}\right)\left(\partial_{t}^{8} v^{i},{ }_{k} A_{i}^{k}\right)=-\frac{1}{2} \frac{d}{d t}\left|\rho_{0} \operatorname{div}_{\eta} \partial_{t}^{7} v\right|^{2}+\frac{1}{2} \rho_{0}^{2} \partial_{t}^{7} v^{r},{ }_{s} \partial_{t}^{7} v^{i},{ }_{k}\left(A_{r}^{s} A_{i}^{k}\right)_{t},
$$

and hence

$$
\mathcal{I}_{1}=\frac{1}{2} \frac{d}{d t} \int_{\Omega} \rho_{0}^{2}\left(J^{-3}\left|D_{\eta} \partial_{t}^{7} v\right|^{2}-J^{-1}\left|\operatorname{curl}_{\eta} \partial_{t}^{7} v\right|^{2}-J^{-1}\left|\operatorname{div}_{\eta} \partial_{t}^{7} v\right|^{2}\right) d x+\mathcal{R} .
$$

It follows that

$$
\begin{aligned}
\int_{0}^{T} \mathcal{I}_{1}(t) d t= & \frac{1}{2} \int_{\Omega} \rho_{0}{ }^{2}\left(\left|D \partial_{t}^{7} v(T)\right|^{2}-J^{-1}\left|\operatorname{curl}_{\eta} \partial_{t}^{7} v(T)\right|^{2}-J^{-1}\left|\operatorname{div}_{\eta} \partial_{t}^{7} v(T)\right|^{2}\right) d x \\
& -M_{0}+\int_{0}^{T} \mathcal{R}(t) d t
\end{aligned}
$$

Analysis of the integral $\mathcal{I}_{2}$. Integration by parts, using (4.5), once again yields

$$
\mathcal{I}_{2}=-\int_{\Omega} \rho_{0}^{2} \partial_{t}^{8} J^{-2} a_{i}^{k} \partial_{t}^{8} v^{i},{ }_{k} d x
$$

Since $\partial_{t}^{8} J^{-2}=-2 J^{-3} \partial_{t}^{8} J$ plus lower-order terms, which have at most seven time derivatives on $J$, and can be estimated in the same fashion as the remainder term $\mathcal{R}$ above.

We see that

$$
\begin{aligned}
\mathcal{I}_{2} & =2 \int_{\Omega} \rho_{0}^{2} J^{-3} a_{s}^{r} \partial_{t}^{7} v^{s},{ }_{r} \quad a_{i}^{k} \partial_{t}^{8} v^{i},{ }_{k} d x+\mathcal{R} \\
& =\frac{d}{d t} \int_{\Omega} \rho_{0}^{2} J^{-3} a_{s}^{r} \partial_{t}^{7} v^{s},{ }_{r} a_{i}^{k} \partial_{t}^{7} v^{i},{ }_{k} d x-\int_{\Omega} \rho_{0}^{2}\left(J^{-3} a_{s}^{r} a_{i}^{k}\right)_{t} \partial_{t}^{7} v^{s},{ }_{r} \partial_{t}^{7} v^{i},{ }_{k} d x+\mathcal{R} .
\end{aligned}
$$

Following our analysis of the term $\mathcal{I}_{2}$ in the $\bar{\partial}^{4}$-problem, we see that

$$
\int_{\Omega} \rho_{0}^{2} J^{-3} a_{s}^{r} \partial_{t}^{7} v^{s}, r \quad a_{i}^{k} \partial_{t}^{7} v^{i},{ }_{k} d x=M_{0}+\int_{0}^{T}\left[\mathcal{I}_{2}(t)+\mathcal{R}(t)\right] d t .
$$

On the other hand,

$$
\begin{aligned}
& \int_{\Omega} \rho_{0}^{2} J^{-3} a_{s}^{r} \partial_{t}^{7} v^{s}, r \quad a_{i}^{k} \partial_{t}^{7} v^{i},{ }_{k} d x \\
& =\int_{\Omega} \rho_{0}^{2} J^{-2}\left(\partial_{t}^{7} \operatorname{div} v+\partial_{t}^{7} v^{s}, r \int_{0}^{t} a_{t_{s}}^{r} d t^{\prime}\right)\left(\partial_{t}^{7} \operatorname{div} v+\partial_{t}^{7} v^{i}, \int_{0}^{t} a_{t i}^{k} d t^{\prime}\right) d x \\
& =\int_{\Omega} \rho_{0}^{2} J^{-2}\left|\partial_{t}^{7} \operatorname{div} v\right|^{2} d x+2 \int_{\Omega} \rho_{0}^{2} J^{-2} \partial_{t}^{7} \operatorname{div} v \partial_{t}^{7} v^{s}, r \int_{0}^{t} a_{t_{s}}^{r} d t^{\prime} d x \\
& \quad+\int_{\Omega} \rho_{0}^{2} J^{-2} \partial_{t}^{7} v^{s}, r \int_{0}^{t} a_{t_{s}}^{r} d t^{\prime} \partial_{t}^{7} v^{i}, \int_{0}^{t} a_{t i}^{k} d t^{\prime} d x
\end{aligned}
$$

Yet another application of the Sobolev embedding theorem together with our assumptions (6.7) and the Cauchy-Schwarz inequality shows that the second and third integrals 
on the right-hand side are bounded by $M_{0}+C \sup _{t \in[0, T]} E(t)$, so that summing (6.26) and (6.27) shows that

$$
\int_{0}^{T} \mathcal{I}_{2}(t) d t=\int_{\Omega} \rho_{0}^{2} J^{-2}\left|\partial_{t}^{7} \operatorname{div} v(T)\right|^{2} d x-M_{0}+\int_{0}^{T} \mathcal{R}(t) d t .
$$

Summing inequalities. We integrate (6.24) from 0 to $T$, and sum (6.10), (6.25), and (6.28) to find that

$$
\begin{aligned}
& \sup _{t \in[0, T]} \frac{1}{2}\left[\int_{\Omega} \rho_{0}^{2} J^{-2}\left|\partial_{t}^{7} D v\right|^{2} d x+\rho_{0}^{2} J^{-2}\left|\partial_{t}^{7} \operatorname{div} v\right|^{2} d x-\int_{\Omega} \rho_{0}^{2} J^{-2}\left|\partial_{t}^{7} \operatorname{curl} v\right|^{2} d x\right] \\
& \quad \leq M_{0}+\delta \sup _{t \in[0, T]} E(t)+C T P\left(\sup _{t \in[0, T]} E(t)\right) .
\end{aligned}
$$

Adding the curl estimate (6.1), readjusting our constants, we obtain the desired result, and complete the proof of the proposition.

6.4. The $\partial_{t}^{2} \bar{\partial}^{3}, \partial_{t}^{4} \bar{\partial}^{2}$, and $\partial_{t}^{6} \bar{\partial}$ problems. Since we have provided detailed proofs of the energy estimates for the two end-point cases of all space derivatives, the $\bar{\partial}^{4}$ problem, and all time derivatives, the $\partial_{t}^{8}$ problem, we have covered all of the estimation strategies for all possible error terms in the three remaining intermediated problems; meanwhile, the energy contributions for the three intermediate are found in the identical fashion as for the $\bar{\partial}^{4}$ and $\partial_{t}^{8}$ problems. As such we have the additional estimate

Proposition 6.5. For $\delta>0$ and letting the constant $M_{0}$ depend on $1 / \delta$, for $\alpha=1,2$,

$$
\begin{aligned}
& \sup _{t \in[0, T]} \sum_{a=1}^{3}\left[\left|\partial_{t}^{2 a} \eta^{\alpha}(t)\right|_{3.5-a}^{2}+\left\|\sqrt{\rho_{0}} \bar{\partial}^{4-a} \partial_{t}^{2 a} v(t)\right\|_{0}^{2}+\left\|\rho_{0} \bar{\partial}^{4-a} \partial_{t}^{2 a} D \eta(t)\right\|_{0}^{2}\right] \\
& \quad \leq M_{0}+\delta \sup _{t \in[0, T]} E(t)+C T P\left(\sup _{t \in[0, T]} E(t)\right) .
\end{aligned}
$$

6.5. Additional elliptic-type estimates for normal derivatives. Our energy estimates provide a priori control of horizontal and time derivatives of $\eta$; it remains to gain a priori control of the normal (or vertical) derivatives of $\eta$. This is accomplished via a bootstrapping procedure relying on having $\partial_{t}^{7} v(t)$ bounded in $L^{2}(\Omega)$.

Proposition 6.6. For $t \in[0, T], \partial_{t}^{5} v(t) \in H^{1}(\Omega), \rho_{0} \partial_{t}^{6} J^{-2}(t) \in H^{1}(\Omega)$ and

$$
\sup _{t \in[0, T]}\left(\left\|\partial_{t}^{5} v(t)\right\|_{1}^{2}+\left\|\rho_{0} \partial_{t}^{6} J^{-2}(t)\right\|_{1}^{2}\right) \leq M_{0}+\delta \sup _{t \in[0, T]} E(t)+C T P\left(\sup _{t \in[0, T]} E(t)\right) .
$$

Proof. We will first assume that

$$
\rho_{0}\left(x_{3}\right)=1-x_{3},
$$

and after establishing our estimates for this particular choice of $\rho_{0}$, we will explain the minor modifications required for the general case of $0<\rho_{0} \in H^{4}(\Omega)$ satisfying (1.5).

We write (1.9a) as $v_{t}^{i}+2 A_{i}^{k}\left(\rho_{0} J^{-1}\right),_{k}=0$, which we rewrite as

$$
v_{t}^{i}+\rho_{0} a_{i}^{k} J^{-2}, k-2 a_{i}^{3} J^{-2}=0 .
$$

We have used the fact that $\rho_{0}, \beta=0$ for $\beta=1,2$, and $\rho_{0,3}=-1$. 
Letting $\partial_{t}^{6}$ act on Eq. (6.29), we have that

$$
\begin{aligned}
\rho_{0} a_{i}^{3} \partial_{t}^{6} J^{-2}, 3-2 a_{i}^{3} \partial_{t}^{6} J^{-2}= & -\partial_{t}^{7} v^{i}-\rho_{0} \partial_{t}^{6}\left(a_{i}^{\beta} J^{-2},_{\beta}\right)-\left(\partial_{t}^{6} a_{i}^{3}\right)\left[-2 J^{-2}+\rho_{0} J^{-2},_{3}\right] \\
& +\sum_{a=1}^{5} c_{a} \partial_{t}^{a} a_{i}^{3} \partial_{t}^{6-a}\left[-2 J^{-2}+\rho_{0} J^{-2},{ }_{3}\right] .
\end{aligned}
$$

According to Propositions 6.4 and 6.5,

$$
\sup _{t \in[0, T]}\left(\left\|\partial_{t}^{7} v(t)\right\|_{0}^{2}+\left\|\rho_{0} \bar{\partial} D \partial_{t}^{5} v(t)\right\|_{0}^{2}\right) \leq M_{0}+\delta \sup _{t \in[0, T]} E(t)+C T P\left(\sup _{t \in[0, T]} E(t)\right),
$$

and since (4.6) shows that $a_{i}^{3}$ is quadratic in $\bar{\partial} \eta$, we see that for all $t \in[0, T]$,

$$
\left\|\left[\rho_{0} a_{i}^{3} \partial_{t}^{6} J^{-2}, 3-2 a_{i}^{3} \partial_{t}^{6} J^{-2}\right](t)\right\|_{0}^{2} \leq M_{0}+\delta \sup _{t \in[0, T]} E(t)+C T P\left(\sup _{t \in[0, T]} E(t)\right) .
$$

It follows that

$$
\begin{aligned}
& \left\|\rho_{0}\left|a_{.}^{3}\right| \partial_{t}^{6} J^{-2},{ }_{3}(t)\right\|_{0}^{2}+4\left\|\left|a_{.}^{3}\right| \partial_{t}^{6} J^{-2}(t)\right\|_{0}^{2}-4 \int_{\Omega} \rho_{0}\left|a_{.}^{3}\right|^{2} \partial_{t}^{6} J^{-2} \partial_{t}^{6} J^{-2},_{3} d x \\
& \leq M_{0}+\delta \sup _{t \in[0, T]} E(t)+C T P\left(\sup _{t \in[0, T]} E(t)\right) .
\end{aligned}
$$

We assume that our solution is sufficiently smooth so that $\rho_{0}\left[\left(\partial_{t}^{6} J^{-2}\right)^{2}\right],{ }_{3}$ is well-defined and integrable. As such, we write ${ }^{1}$

$$
\begin{aligned}
& -4 \int_{\Omega} \rho_{0}\left|a^{3}\right|^{2} \partial_{t}^{6} J^{-2} \partial_{t}^{6} J^{-2},{ }_{3} d x=-2\left\|\left|a^{3} \cdot\right| \partial_{t}^{6} J^{-2}(t)\right\|_{0}^{2}+2 \int_{\Omega} \rho_{0}\left(\left|a^{3}\right|^{2}\right),,_{3}\left(\partial_{t}^{6} J^{-2}\right)^{2} d x \\
& +4 \int_{\left\{x_{3}=0\right\}}\left|\partial_{t}^{6} J^{-2}\right|^{2} d x_{1} d x_{2},
\end{aligned}
$$

so that together with our previous inequality,

$$
\begin{aligned}
& \left\|\rho_{0} \partial_{t}^{6} J^{-2},_{3}(t)\right\|_{0}^{2}+\left\|\partial_{t}^{6} J^{-2}(t)\right\|_{0}^{2} \\
& \quad \leq M_{0}+\delta \sup _{t \in[0, T]} E(t)+C T P\left(\sup _{t \in[0, T]} E(t)\right)+C \int_{\Omega} \rho_{0}\left|\partial_{t}^{6} J^{-2}\right|^{2} d x .
\end{aligned}
$$

Since $\rho_{0} \bar{\partial} \partial_{t}^{6} J^{-2}(t)$ is already estimated by Proposition 6.5, then

$$
\begin{aligned}
& \left\|\rho_{0} \partial_{t}^{6} J^{-2}(t)\right\|_{1}^{2}+\left\|\partial_{t}^{6} J^{-2}(t)\right\|_{0}^{2} \\
& \quad \leq M_{0}+\delta \sup _{t \in[0, T]} E(t)+C T P\left(\sup _{t \in[0, T]} E(t)\right)+C \int_{\Omega} \rho_{0}\left|\partial_{t}^{6} J^{-2}\right|^{2} d x .
\end{aligned}
$$

\footnotetext{
1 Jang \& Masmoudi [7] have counterexamples to the obtained inequality when $J^{-2}$ is not sufficiently smooth. It is important that the function $J^{-2}$ has greater regularity than the desired a priori estimate indicates, and in particular, as we noted, $\rho_{0}\left[\left(\partial_{t}^{6} J^{-2}\right)^{2}\right], 3$ must be well-defined and integrable.
} 
We use Young's inequality and the fundamental theorem of calculus (with respect to $t$ ) for the last integral to find that for $\delta>0$,

$$
\begin{aligned}
C \int_{\Omega^{+}} \rho_{0} \partial_{t}^{6} J^{-2} \partial_{t}^{6} J^{-2} d x \leq & \delta\left\|\partial_{t}^{6} J^{-2}(t)\right\|_{0}^{2}+C_{\delta}\left\|\rho_{0} \partial_{t}^{5} D v(t)\right\|_{0}^{2} \\
& +M_{0}+C T P\left(\sup _{t \in[0, T]} E(t)\right) \\
\leq & \delta\left\|\partial_{t}^{6} J^{-2}(t)\right\|_{0}^{2}+M_{0}+C T P\left(\sup _{t \in[0, T]} E(t)\right),
\end{aligned}
$$

where we have used the fact that $\left\|\rho_{0} \partial_{t}^{7} D v(t)\right\|_{0}^{2}$ is contained in the energy function $E(t)$. By once again readjusting the constants, we see that on $[0, T]$,

$$
\left\|\rho_{0} \partial_{t}^{6} J^{-2}(t)\right\|_{1}^{2}+\left\|\partial_{t}^{6} J^{-2}(t)\right\|_{0}^{2} \leq M_{0}+\delta \sup _{t \in[0, T]} E(t)+C T P\left(\sup _{t \in[0, T]} E(t)\right) .
$$

With $J_{t}=a_{i}^{j} v^{i},{ }_{j}$, we see that

$$
a_{i}^{j} \partial_{t}^{5} v_{j}^{i}=\partial_{t}^{6} J-v^{i},{ }_{j} \partial_{t}^{5} a_{i}^{j}-\sum_{a=1}^{4} c_{a} \partial_{t}^{a} a_{i}^{j} \partial_{t}^{5-a} v^{i},{ }_{j},
$$

so that using (6.30) together with the fundamental theorem of calculus the estimate for the last two terms on the right-hand side, we see that

$$
\left\|a_{i}^{j} \partial_{t}^{5} v^{i},{ }_{j}(t)\right\|_{0}^{2} \leq M_{0}+\delta \sup _{t \in[0, T]} E(t)+C T P\left(\sup _{t \in[0, T]} E(t)\right),
$$

from which it follows that

$$
\left\|\operatorname{div} \partial_{t}^{5} v(t)\right\|_{0}^{2} \leq M_{0}+\delta \sup _{t \in[0, T]} E(t)+C T P\left(\sup _{t \in[0, T]} E(t)\right) .
$$

According to Proposition 6.1, $\left\|\operatorname{curl} \partial_{t}^{5} v(t)\right\|_{0}^{2} \leq M_{0}+C T P\left(\sup _{t \in[0, T]} E(t)\right)$ and with the bound on $\partial_{t}^{5} v^{\alpha}$ given by Proposition 6.5, Proposition 5.2 provides the estimate

$$
\left\|\partial_{t}^{5} v(t)\right\|_{1}^{2} \leq M_{0}+\delta \sup _{t \in[0, T]} E(t)+C T P\left(\sup _{t \in[0, T]} E(t)\right) .
$$

More generally, for any $0<\rho_{0} \in H^{4}(\Omega)$ satisfying (1.5), Eq. (6.29) takes the form, for $\beta=1,2$,

$$
v_{t}^{i}+\rho_{0} a_{i}^{k} J^{-2},{ }_{k}+2 \rho_{0,3} a_{i}^{3} J^{-2}+2 \rho_{0}, \beta a_{i}^{\beta} J^{-2}=0 .
$$

Letting $\partial_{t}^{6}$ act on this equation yields

$$
\begin{aligned}
& \rho_{0} a_{i}^{3} \partial_{t}^{6} J^{-2},{ }_{3}+2 \rho_{0,3} a_{i}^{3} \partial_{t}^{6} J^{-2}=-\partial_{t}^{7} v^{i}-\rho_{0} \partial_{t}^{6}\left(a_{i}^{\beta} J^{-2}, \beta\right)-2 \rho_{0}, \beta \partial_{t}^{6}\left(a_{i}^{\beta} J^{-2}\right) \\
& -\left(\partial_{t}^{6} a_{i}^{3}\right)\left[\rho_{0} J^{-2}, 3+2 \rho_{0}, 3 J^{-2}\right]+\sum_{a=1}^{5} c_{a} \partial_{t}^{a} a_{i}^{3} \partial_{t}^{6-a}\left[\rho_{0} J^{-2},{ }_{3}+2 \rho_{0},{ }_{3} J^{-2}\right] \text {. }
\end{aligned}
$$


As such, if the $L^{2}(\Omega)$-norm of the right-hand side of (6.31) is bounded by $M_{0}+$ $\delta \sup _{t \in[0, T]} E(t)+C T P\left(\sup _{t \in[0, T]} E(t)\right)$, then the identical argument detailed above would lead to the inequality

$$
\left\|\rho_{0} \partial_{t}^{6} J^{-2}(t)\right\|_{1}^{2}+\left\|\left|\rho_{0}, 3\right| \partial_{t}^{6} J^{-2}(t)\right\|_{0}^{2} \leq M_{0}+\delta \sup _{t \in[0, T]} E(t)+C T P\left(\sup _{t \in[0, T]} E(t)\right) .
$$

By the physical vacuum condition (1.5), for $\epsilon>0$ taken sufficiently small, there are constants $\theta_{1}, \theta_{2}>0$ such that $\left|\rho_{0}, 3(x)\right| \geq \theta_{1}$ whenever $1-\epsilon \leq x_{3} \leq 1$, and $\rho_{0}(x)>\theta_{2}$ whenever $0 \leq x \leq 1-\epsilon$; hence, by readjusting the constants, we obtain the inequality (6.30).

As the $L^{2}(\Omega)$-bound for the right-hand side of (6.31), the only new type of term that the general function $\rho_{0}$ produces is $-2 \rho_{0}, \beta \partial_{t}^{6}\left(a_{i}^{\beta} J^{-2}\right)$. On the other hand, given that $\rho_{0} \in H^{4}(\Omega)$ and that $\rho_{0}=0$ on $\Gamma$, the Sobolev embedding theorem shows that for $\beta=$ $1,2,\left\|\rho_{0}, \beta / \rho_{0}\right\|_{L^{\infty}(\Omega)} \leq C\left\|\rho_{0}, \beta / \rho_{0}\right\|_{2} \leq\left\|\rho_{0}\right\|_{4}<C$, so that $\left|\rho_{0}, \beta(x)\right| \leq C \rho_{0}(x)$. This shows that

$$
\begin{aligned}
& \left\|2 \rho_{0}, \beta \partial_{t}^{6}\left(a_{i}^{\beta} J^{-2}\right)\right\|_{0}^{2} \leq C\left\|2 \rho_{0} \partial_{t}^{6}\left(\left[a_{i}^{1}+a_{i}^{2}\right] J^{-2}\right)\right\|_{0}^{2} \\
& \leq M_{0}+\delta \sup _{t \in[0, T]} E(t)+C T P\left(\sup _{t \in[0, T]} E(t)\right) \text {. }
\end{aligned}
$$

Having a good bound for $\partial_{t}^{5} v(t)$ in $H^{1}(\Omega)$ we proceed with our bootstrapping.

Proposition 6.7. For $t \in[0, T], v_{t t t}(t) \in H^{2}(\Omega), \rho_{0} \partial_{t}^{4} J^{-2}(t) \in H^{2}(\Omega)$ and

$$
\sup _{t \in[0, T]}\left(\left\|v_{t t t}(t)\right\|_{2}^{2}+\left\|\rho_{0} \partial_{t}^{4} J^{-2}(t)\right\|_{2}^{2}\right) \leq M_{0}+\delta \sup _{t \in[0, T]} E(t)+C T P\left(\sup _{t \in[0, T]} E(t)\right) .
$$

Proof. We let $\partial_{t}^{4}$ act on Eq. (6.29), and using the argument just given above, it suffices to consider the case that $\rho_{0}=1-x_{3}$. It follows that

$$
\begin{aligned}
\rho_{0} a_{i}^{3} \partial_{t}^{4} J^{-2}, 3-2 a_{i}^{3} \partial_{t}^{4} J^{-2}= & -\partial_{t}^{5} v^{i}-\rho_{0} \partial_{t}^{4}\left(a_{i}^{\beta} J^{-2}, \beta\right)-\left(\partial_{t}^{4} a_{i}^{3}\right)\left[-2 J^{-2}+\rho_{0} J^{-2},_{3}\right] \\
& +\sum_{a=1}^{3} c_{a} \partial_{t}^{a} a_{i}^{3} \partial_{t}^{4-a}\left[-2 J^{-2}+\rho_{0} J^{-2},{ }_{3}\right] .
\end{aligned}
$$

In order to estimate $\partial_{t}^{4} J^{-2}(t)$ in $H^{1}(\Omega)$, we first estimate horizontal derivatives of $\partial_{t}^{4} J^{-2}(t)$ in $L^{2}(\Omega)$. As such, we consider for $\alpha=1,2$,

$$
\begin{aligned}
\rho_{0} a_{i}^{3} \partial_{t}^{4} J^{-2}, 3 \alpha-2 a_{i}^{3} \partial_{t}^{4} J^{-2}, \alpha= & {\left[-\partial_{t}^{5} v^{i}-\rho_{0} \partial_{t}^{4}\left(a_{i}^{\beta} J^{-2}, \beta\right)-\left(\partial_{t}^{4} a_{i}^{3}\right)\left(-2 J^{-2}\right.\right.} \\
& \left.\left.+\rho_{0} J^{-2},_{3}\right)+\sum_{a=1}^{3} c_{a} \partial_{t}^{a} a_{i}^{3} \partial_{t}^{4-a}\left(-2 J^{-2}+\rho_{0} J^{-2},{ }_{3}\right)\right],{ }_{\alpha} \\
& -\rho_{0} a_{i}^{3},_{\alpha} \partial_{t}^{4} J^{-2},_{3}+2 a_{i}^{3},{ }_{\alpha} \partial_{t}^{4} J^{-2} .
\end{aligned}
$$


According to Proposition 6.5, the right-hand side of (6.34) is bounded in $L^{2}(\Omega)$ by $M_{0}+\delta \sup _{t \in[0, T]} E(t)+C T P\left(\sup _{t \in[0, T]} E(t)\right)$. Using the argument just given above in the proof of Proposition 6.6, we conclude that for $\alpha=1,2$,

$$
\sup _{t \in[0, T]}\left(\left\|v_{t t t}, \alpha(t)\right\|_{1}^{2}+\left\|\rho_{0} \partial_{t}^{4} J^{-2}, \alpha(t)\right\|_{1}^{2}\right) \leq M_{0}+\delta \sup _{t \in[0, T]} E(t)+C T P\left(\sup _{t \in[0, T]} E(t)\right) .
$$

We next differentiate (6.33) in the vertical direction $x_{3}$ to obtain

$$
\begin{aligned}
\rho_{0} a_{i}^{3} \partial_{t}^{4} J^{-2},_{33}-3 a_{i}^{3} \partial_{t}^{4} J^{-2},_{3}= & {\left[-\partial_{t}^{5} v^{i}-\rho_{0} \partial_{t}^{4}\left(a_{i}^{\beta} J^{-2}, \beta\right)-\left(\partial_{t}^{4} a_{i}^{3}\right)\left(-2 J^{-2}+\rho_{0} J^{-2},{ }_{3}\right)\right.} \\
& \left.+\sum_{a=1}^{3} c_{a} \partial_{t}^{a} a_{i}^{3} \partial_{t}^{4-a}\left(-2 J^{-2}+\rho_{0} J^{-2},{ }_{3}\right)\right], 3 \\
& -\rho_{0} a_{i}^{3},_{3} \partial_{t}^{4} J^{-2},{ }_{3}+2 a_{i}^{3},{ }_{3} \partial_{t}^{4} J^{-2} .
\end{aligned}
$$

Now the inequality (6.35) together with Propositions 6.5 and 6.6 show that the right-hand side of (6.36) is bounded in $L^{2}(\Omega)$ by $M_{0}+\delta \sup _{t \in[0, T]} E(t)+C T P\left(\sup _{t \in[0, T]} E(t)\right)$.

It follows that for $k=1,2,3$,

$$
\left\|\rho_{0} a_{i}^{3} \partial_{t}^{4} J^{-2}, k 3-3 a_{i}^{3} \partial_{t}^{4} J^{-2},{ }_{k}\right\|_{0}^{2} \leq M_{0}+\delta \sup _{t \in[0, T]} E(t)+C T P\left(\sup _{t \in[0, T]} E(t)\right) .
$$

Note that the coefficient in front of $a_{i}^{3} \partial_{t}^{4} J^{-2}$ has changed from -2 to -3 , but the identical integration-by-parts argument that we used in the proof of Proposition 6.6 is once again employed and shows that

$$
\left\|\rho_{0} \partial_{t}^{4} J^{-2}(t)\right\|_{2}^{2}+\left\|\partial_{t}^{4} J^{-2}(t)\right\|_{1}^{2} \leq M_{0}+\delta \sup _{t \in[0, T]} E(t)+C T P\left(\sup _{t \in[0, T]} E(t)\right) .
$$

We can thus infer that

$$
\left\|\operatorname{div} v_{t t t}(t)\right\|_{1}^{2} \leq M_{0}+\delta \sup _{t \in[0, T]} E(t)+C T P\left(\sup _{t \in[0, T]} E(t)\right) .
$$

According to Proposition 6.1, $\left\|\operatorname{curl} v_{t t t}(t)\right\|_{1}^{2} \leq M_{0}+C T P\left(\sup _{t \in[0, T]} E(t)\right)$ and with the bound on $v_{t t t}^{\alpha}$ given by Proposition 6.5, Proposition 5.2 provides the estimate

$$
\left\|v_{t t t}(t)\right\|_{2}^{2} \leq M_{0}+\delta \sup _{t \in[0, T]} E(t)+C T P\left(\sup _{t \in[0, T]} E(t)\right) .
$$

Proposition 6.8. For $t \in[0, T], v_{t}(t) \in H^{3}(\Omega), \rho_{0} \partial_{t}^{2} J^{-2}(t) \in H^{3}(\Omega)$ and

$$
\sup _{t \in[0, T]}\left(\left\|v_{t}(t)\right\|_{3}^{2}+\left\|\rho_{0} \partial_{t}^{2} J^{-2}(t)\right\|_{3}^{2}\right) \leq M_{0}+\delta \sup _{t \in[0, T]} E(t)+C T P\left(\sup _{t \in[0, T]} E(t)\right) .
$$


Proof. Next, we let $\partial_{t}^{2}$ act on Eq. (6.29), so that

$$
\begin{aligned}
\rho_{0} a_{i}^{3} \partial_{t}^{2} J^{-2},{ }_{3}-2 a_{i}^{3} \partial_{t}^{2} J^{-2}= & -\partial_{t}^{3} v^{i}-\rho_{0} \partial_{t}^{2}\left(a_{i}^{\beta} J^{-2}, \beta\right)-\left(\partial_{t}^{2} a_{i}^{3}\right)\left[-2 J^{-2}+\rho_{0} J^{-2},{ }_{3}\right] \\
& +2\left(\partial_{t} a_{i}^{3}\right) \partial_{t}\left[-2 J^{-2}+\rho_{0} J^{-2},,_{3}\right] .
\end{aligned}
$$

The same argument used in the proof of Proposition 6.7 provides the desired inequality.

Proposition 6.9. For $t \in[0, T], \eta(t) \in H^{4}(\Omega), \rho_{0} J^{-2}(t) \in H^{4}(\Omega)$ and

$$
\sup _{t \in[0, T]}\left(\|\eta(t)\|_{4}^{2}+\left\|\rho_{0} J^{-2}(t)\right\|_{4}^{2}\right) \leq M_{0}+\delta \sup _{t \in[0, T]} E(t)+C T P\left(\sup _{t \in[0, T]} E(t)\right) .
$$

Proof. We use the identity

$$
\rho_{0} a_{i}^{3} J^{-2}, 3-2 a_{i}^{3} J^{-2}=-v_{t}^{i}-\rho_{0} a_{i}^{\beta} J^{-2}, \beta .
$$

The same argument used in the proof of Proposition 6.7 provides the desired inequality.

6.6. Estimates for $\operatorname{curl}_{\eta} v$. The regularity for the Lagrangian curl of $v$ gains regularity.

\section{Corollary 6.10.}

$$
\sup _{t \in[0, T]}\left(\left\|\operatorname{curl}_{\eta} v(t)\right\|_{3}^{2}+\left\|\rho_{0} \bar{\partial}^{4} \operatorname{curl}_{\eta} v(t)\right\|_{0}^{2}\right) \leq M_{0}+\delta \sup _{t \in[0, T]} E(t)+C T P\left(\sup _{t \in[0, T]} E(t)\right) .
$$

Proof. Letting $D^{3}$ act on the identity (6.2) for $\operatorname{curl}_{\eta} v$, we see that the highest-order term scales like

$$
D^{3} \operatorname{curl} u_{0}+\int_{0}^{t} D^{4} v D v A A d t^{\prime}
$$

We integrate by parts to see that the highest-order contribution to $D^{3} \operatorname{curl}_{\eta} v(t)$ can be written as

$$
D^{3} \operatorname{curl} u_{0}-\int_{0}^{t} D^{4} \eta[D v A A]_{t} d t^{\prime}+D^{4} \eta(t) D v(t) A(t) A(t)
$$

which, according to Proposition 6.9, has $L^{2}(\Omega)$-norm bounded by

$$
M_{0}(\delta)+\delta \sup _{t \in[0, T]} E(t)+C T P\left(\sup _{t \in[0, T]} E(t)\right),
$$

after readjusting the constants; thus, the inequality for the $H^{3}(\Omega)$-norm of $\operatorname{curl}_{\eta} v(t)$ is proved.

The same type of analysis works for the weighted estimate. After integration by parts in time, the highest-order term in the expression for $\rho_{0} \bar{\partial}^{4} \operatorname{curl}_{\eta} v(t)$ scales like

$$
\rho_{0} \bar{\partial}^{4} \operatorname{curl} u_{0}-\int_{0}^{t} \rho_{0} \bar{\partial}^{4} D \eta[D v A A]_{t} d t^{\prime}+\rho_{0} \bar{\partial}^{4} D \eta(t) D v(t) A(t) A(t) .
$$

Hence, the inequality (6.8) shows that the weighted estimate holds as well. 
6.7. The a priori bound. Summing the inequalities provided by our energy estimates, the additional elliptic estimates, and the estimates for $\operatorname{curl}_{\eta} v$ shows that

$$
\sup _{t \in[0, T]} E(t) \leq M_{0}+C T P\left(\sup _{t \in[0, T]} E(t)\right) .
$$

According to our polynomial-type inequality given in Sect. 4.5 , by taking $T>0$ sufficiently small, we have the a priori bound

$$
\sup _{t \in[0, T]} E(t) \leq 2 M_{0}
$$

\section{The Case of General $\gamma>1$}

We denote by $a_{0}$ the integer satisfying the inequality

$$
1<1+\frac{1}{\gamma-1}-a_{0} \leq 2 .
$$

The general higher-order energy function is given by

$$
\begin{aligned}
E_{\gamma}(t)= & \sum_{a=0}^{4}\left\|\partial_{t}^{2 a} \eta(t)\right\|_{4-a}^{2}+\sum_{a=0}^{4}\left[\left\|\rho_{0} \bar{\partial}^{4-a} \partial_{t}^{2 a} D \eta(t)\right\|_{0}^{2}+\left\|\sqrt{\rho_{0}} \bar{\partial}^{4-a} \partial_{t}^{2 a} v(t)\right\|_{0}^{2}\right] \\
& +\sum_{a=0}^{3}\left\|\rho_{0} \partial_{t}^{2 a} J^{-2}(t)\right\|_{4-a}^{2}+\left\|\operatorname{curl}_{\eta} v(t)\right\|_{3}^{2}+\left\|\rho_{0} \bar{\partial}^{4} \operatorname{curl}_{\eta} v(t)\right\|_{0}^{2} \\
& +\sum_{a=0}^{a_{0}}\left\|{\sqrt{\rho_{0}}}^{1+\frac{1}{\gamma-1}}-a \partial_{t}^{7+a_{0}-a} D v(t)\right\|_{0}^{2} .
\end{aligned}
$$

Notice the last sum in $E_{\gamma}$ appears whenever $\gamma<2$, and the number of time-differentiated problems increases as $\gamma$ approaches 1 . Using this energy function, the same methodology as we used for the case $\gamma=2$, shows that $\sup _{t \in[0, T]} E_{\gamma}(t)$ remains bounded for $T>0$ taken sufficiently small.

Acknowledgements. We thank the referee for useful suggestions which have improved the manuscript. SS was supported by the National Science Foundation under grant DMS-0701056. HL was supported by the National Science Foundation under grant DMS-0801120.

Open Access This article is distributed under the terms of the Creative Commons Attribution Noncommercial License which permits any noncommercial use, distribution, and reproduction in any medium, provided the original author(s) and source are credited.

\section{References}

1. Cheng, A., Coutand, D., Shkoller, S.: On the Motion of Vortex Sheets with Surface Tension in the 3D Euler Equations with Vorticity. Comm. Pure Appl. Math. 61, 1715-1752 (2008)

2. Coutand, D., Shkoller, S.: On the interaction between quasilinear elastodynamics and the Navier-Stokes equations. Arch. Rat. Mech. Anal. 179(3), 303-352 (2006)

3. Coutand, D., Shkoller, S.: Well-posedness of the free-surface incompressible Euler equations with or without surface tension. J. Amer. Math. Soc. 20, 829-930 (2007)

4. Coutand, D., Lindblad, H., Shkoller, S.: 2007 SIAM Conference on Analysis of Partial Differential Equations, Dec. 10, 2007 
5. Evans, L.C.: Partial differential equations. Graduate Studies in Mathematics, 19. Providence, RI: Amer. Math. Soc., 1998

6. Jang, J., Masmoudi, N.: Well-posedness for compressible Euler with physical vacuum singularity. Comm. Pure Appl. Math. 62, 1327-1385 (2009)

7. Private communication with Steve Shkoller on Oct. 7, 2008 at NYU

8. Kreiss, H.O.: Initial boundary value problems for hyperbolic systems. Commun. Pure Appl. Math. 23, 277-296 (1970)

9. Kufner, A.: Weighted Sobolev Spaces. New York: Wiley-Interscience, 1985

10. Lin, L.W.: On the vacuum state for the equations of isentropic gas dynamics. J. Math. Anal. Appl. 121, 406-425 (1987)

11. Lindblad, H.: Well posedness for the motion of a compressible liquid with free surface boundary. Commun. Math. Phys. 260, 319-392 (2005)

12. Liu, T.-P.: Compressible flow with damping and vacuum. Japan J. Appl. Math. 13, 25-32 (1996)

13. Liu, T.-P., Yang, T.: Compressible Euler equations with vacuum. J. Diff. Eqs. 140, 223-237 (1997)

14. Liu, T.-P., Yang, T.: Compressible flow with vacuum and physical singularity. Meth. Appl. Anal. 7, 495-510 (2000)

15. Liu, T.-P., Smoller, J.: On the vacuum state for isentropic gas dynamics equations. Adv. Math. 1, 345-359 (1980)

16. Makino, T.: On a local existence theorem for the evolution equation of gaseous stars. In: Patterns and waves, Stud. Math. Appl. 18, Amsterdam: North-Holland, 1986, pp. 459-479

17. Taylor, M.: Partial Differential Equations, Vol. I-III, Berlin-Heidelberg-New York: Springer, 1996

18. Temam, R.: Navier-Stokes equations. Theory and Numerical Analysis. Third edition. Studies in Mathematics and its Applications 2. Amsterdam: North-Holland Publishing Co., 1984

19. Trakhinin, Y.: Local existence for the free boundary problem for the non-relativistic and relativistic compressible Euler equations with a vacuum boundary condition. http://arXiv.org/abs/0810.2612v2[math. AP], 2009

20. Xu, C.-J., Yang, T.: Local existence with physical vacuum boundary condition to Euler equations with damping. J. Diff. Eqs. 210, 217-231 (2005)

Communicated by P. Constantin 